\title{
EFFECT OF TIME DELAY ON A DETRITUS-BASED ECOSYSTEM
}

\author{
NURUL HUDA GAZI AND MALAY BANDYOPADHYAY \\ Received 22 April 2005; Revised 15 July 2006; Accepted 18 July 2006
}

Models of detritus-based ecosystems with delay have received a great deal of attention for the last few decades. This paper deals with the dynamical analysis of a nonlinear model of a detritus-based ecosystem involving detritivores and predator of detritivores. We have obtained the criteria for local stability of various equilibrium points and persistence of the model system. Next, we have introduced discrete time delay due to recycling of dead organic matters and gestation of nutrients to the growth equations of various trophic levels. With delay differential equation model system we have studied the effect of time delay on the stability behaviour. Next, we have obtained an estimate for the length of time delay to preserve the stability of the model system. Finally, the existence of Hopfbifurcating small amplitude periodic solutions is derived by considering time delay as a bifurcation parameter.

Copyright @ 2006 Hindawi Publishing Corporation. All rights reserved.

\section{Introduction}

The dynamical theory of population biology has started to take a formal shape after the pioneering work of Jorgensen [19]. The early studies of small mammals and laboratory controlled organisms involve the interest of mathematician with the dynamics of the population. Our interest here involves in studying the detritus-based ecosystem mainly found in India's Sunderban Mangrove area. The mangrove ecosystem consists of several basic subsystems connected with each other. This ecosystem comprises of many sand flats and mud. Some algal species are always found in these sand flats and mud. They always contribute some amount of detritus which is formed by the action of decomposers on dead bodies of these species. At the primary level the major energy source is the leaves of the mangrove trees which are the nutrient to the higher trophic levels [5]. The supralittoral zones of this ecosystem are occupied by large number of mangrove plants and the plant leaves are chief source of detritus. In this food chain some 10 percent or less of net primary production is grazed and 90 percent passes through decomposers [8]. The food resource of mangrove trees is consumed by small animals such as microarthropods, oligochaetes,

Hindawi Publishing Corporation

International Journal of Mathematics and Mathematical Sciences

Volume 2006, Article ID 25619, Pages 1-28

DOI 10.1155/IJMMS/2006/25619 
and micro-organisms like protozoa, fungi, actinomycetes, bacteria, and so forth. These are considered as the detritivores. The abundance of this detritivores pool solely governs abundance and persistence of invertebrates, namely, nematodes, unicellular animals, and certain insect larvae $[18,31]$. These are known as predators of detritivores. This type of food chains is observed within every ecosystem and is very important for the circulation of materials. The detritus is supplied by the action of decomposers on dead bodies of the algal macrophytes present within the environment and this detritus provides food and refuge for detritivores like amphipod and leptostracan crustaceans. These types of detritus-based models are studied by Vetter [34] for the Southern California Submarine canyon ecosystem. Faust and Gulledge [10] investigated the formation of detritus from microalgae and its association with meiofauna such as nematodes, ciliales copepods, and crustacean larvae at a mangrove island. Charles [5] pointed out that detritus is formed by the action of two types of macrophytes, namely, Cytoseira mediteranca and Posidonia oceanica and the role of detritivores is played by Abra ovata. Linley [21], Ray and Choudhury [29], and Linley and Adams [22] have established that the main source of detritus in the supralittoral zone of mangrove ecosystem in Sunderban is not algae, which is mainly formed by the excessive mortality rate of insect intruders, particularly, in the larval and pupal stages due to high salinity of the trapped water in the supralittoral zone. Consequently, the flow of dead organic matter is not always continuous and is maximum in the intermediate period between a new moon and a full moon. Detritivores depend upon the detritus for food as well as energy and these detritivores also support a novel food source for fish, insect intruders present within the supralittoral zones. There has been considerable interest in mathematical models simulating the interaction between species and decomposers. Sarkar et al. [32] studied the stability of partially closed producer-consumer system via decomposer. In another work, they have studied the persistence and oscillations of the detritus-based prey-predator model and have found that the food conversion efficiency rate of microbial organisms governs the dynamics of such a model.

The time delays of one type on another work was recognized by Volterra [35] and it has been incorporated into biological models by many researchers, namely, Cushing [6], Gopalsamy [15], Kuang [20], and MacDonald [23]. For the prey-predator model, the delay has been studied by Bandyopadhyay [1], Beretta and Kuang [3], Gopalsamy [13, 14], Hastings [17], Martin and Ruan [25], May [26], Ruan [30], and the references cited therein. In most of the cases, population of one species does not respond instantaneously with the other species present in the community. It is believed that the time delays have destabilizing effect on the models of population dynamics and time delays are responsible for population oscillations within deterministic environment.

In this paper we have considered a deterministic model depicting the interaction between the micro-organism pool living on mangrove litter and their invertebrate predators. The growth equation of the detritus has a constant input rate maintained by large macrophytes and algal flora. A part of dead organic matter is converted into detritus by the action of micro-organisms. The consumption of detritus by micro-organism biomass (detritivores) is assumed to follow Holling type-II functional response. Also, the amount of detritivores biomass consumed by its predator follows Holling type-II functional response. In our study we have taken into account the instantaneous constant supply of 
the detritus. In nature, certain days are required to form detritus from the dead organic matter and hence we have considered a time delay due to recycle in the growth equation of detritus. Also, in the growth equation of the detritivores we have considered time delay in the functional response term for gestation due to the consumption of detritus by the detritivores and same type of gestation delay is introduced in the growth equation of the predator of detritivores due to the consumption of detritivores by the predators of detritivores. We study the local stability in presence of time delay and also in absence of time delay. Then we have obtained an estimate of time delay for which the stability behaviour remains unaltered. Finally, we have derived the condition for the existence of Hopf-bifurcating periodic solutions by considering the time delay as bifurcation parameter.

\section{Basic model}

Let $N_{1}=N_{1}(t)$ represent biomass of plant litter from the mangrove trees after decomposition which we call detritus, let $N_{2}=N_{2}(t)$ be the biomass of micro-organisms, namely, detritivores and the biomass of the predators of detritivores be represented by $N_{3}=N_{3}(t)$ which are the invertebrate predators living on micro-organisms at time " $t$." Here we assume that the state variables are nutrient equivalent and the units are same as that of detritus. It is assumed that the growth equation of the detritus is governed by a nonlinear ordinary differential equation

$$
\frac{d N_{1}}{d t}=N_{0}-a N_{1}-\alpha \frac{N_{1} N_{2}}{\beta+N_{1}}
$$

where $N_{0}$ is the constant supply rate of detritus within the system. We assume the loss of detritus biomass due to that leaching is " $a N_{1}$." The loss of detritus biomass due to the consumption by the detritivores follows the Holling type-II functional response, $\alpha$ is the uptake rate and $\beta$ is half saturation constant. The growth equation of the detritivores is governed by

$$
\frac{d N_{2}}{d t}=\alpha_{1} \frac{N_{1} N_{2}}{\beta+N_{1}}-\gamma N_{2}-\delta \frac{N_{2} N_{3}}{\mu+N_{2}}
$$

Let $e_{1}\left(0<e_{1}<1\right)$ be the consumption rate of detritus by detritivores and hence the growth rate of detritivores due to consumption of the detritus is $\alpha_{1}=e_{1} \alpha<\alpha$. Clearly, the rate at which detritus population decreases is higher than the growth rate of detritivores. This is due to the conversion efficiency of detritivores and the coefficient of efficiency is given by $\alpha_{1} / \alpha$. In detritivores growth equation $\gamma$ is the natural mortality rate of detritivores, $\delta$ is the loss rate of detritivores biomass due to the uptake by the invertebrate predators $N_{3}, \mu$ parametrize the half saturation of Holling type-II functional response for the detritivores. The growth rate of predator of detritivores is given by

$$
\frac{d N_{3}}{d t}=\delta_{1} \frac{N_{2} N_{3}}{\mu+N_{2}}-\gamma_{1} N_{3}
$$


where $\delta_{1}$ is the rate of input to the predator of detritivores population due to consumption of detritivores and $\gamma_{1}$ is the natural mortality rate of predator of detritivores. With the similar argument as above, we obtain $\delta_{1}<\delta$.

Finally, we assume that the dead micro-organism whose biomass is $\gamma \mathrm{N}_{2}$ contributes to the growth of the detritus. Then the growth equation (2.1) of the detritus is given by

$$
\frac{d N_{1}}{d t}=N_{0}-a N_{1}-\alpha \frac{N_{1} N_{2}}{\beta+N_{1}}+c \gamma N_{2}
$$

where " $c$ " $(0<c \ll 1)$ and " $c \gamma$ " is the detritus recycle rate after the death of detritivores. Thus our mathematical model takes its formal shape as

$$
\begin{gathered}
\frac{d N_{1}}{d t}=N_{0}-a N_{1}-\alpha \frac{N_{1} N_{2}}{\beta+N_{1}}+c \gamma N_{2}, \\
\frac{d N_{2}}{d t}=\alpha_{1} \frac{N_{1} N_{2}}{\beta+N_{1}}-\gamma N_{2}-\delta \frac{N_{2} N_{3}}{\mu+N_{2}}, \\
\frac{d N_{3}}{d t}=\delta_{1} \frac{N_{2} N_{3}}{\mu+N_{2}}-\gamma_{1} N_{3} .
\end{gathered}
$$

Nonnegative initial conditions are given by $N_{1}(0)=N_{10}>0, N_{2}(0)=N_{20}>0, N_{3}(0)=$ $N_{30}>0$.

\section{Equilibria and boundedness of the model}

We concentrate ourselves to the model system (2.5). The right-hand sides of the system (2.5) are smooth functions of the biomass $N_{1}, N_{2}, N_{3}$ with the initial condition mentioned as above. The parameters involved with the model system are all nonnegative. The equilibria or steady states are the nonnegative solutions of the system of equations

$$
\frac{d N_{1}}{d t}=0, \quad \frac{d N_{2}}{d t}=0, \quad \frac{d N_{3}}{d t}=0
$$

Solving these equations one can obtain the following equilibrium points:

(1) axial equilibrium: $E_{1} \equiv\left(N_{0} / a, 0,0\right)$;

(2) boundary equilibrium: $E_{2} \equiv\left(N_{1 b}, N_{2 b}, 0\right)$, where $N_{1 b}=\beta \gamma /\left(\alpha_{1}-\gamma\right), N_{2 b}=(1 /$ $\left.\gamma\left(\alpha / \alpha_{1}-c\right)\right)\left(N_{0}-a \beta \gamma /\left(\alpha_{1}-\gamma\right)\right)$

(3) interior equilibrium: $E_{*} \equiv\left(N_{1 *}, N_{2 *}, N_{3 *}\right)$, where $N_{1 *}, N_{2 *}, N_{3 *}$ are given by

$$
\begin{gathered}
N_{1 *}=\frac{1}{2 a}\left[N_{0}+\frac{c \mu \gamma \gamma_{1}}{\delta_{1}-\gamma_{1}}-a \beta-\frac{\alpha \mu \gamma_{1}}{\delta_{1}-\gamma_{1}}\right. \\
\quad+\sqrt{\left.\left(N_{0}+\frac{c \mu \gamma \gamma_{1}}{\delta_{1}-\gamma_{1}}-a \beta-\frac{\alpha \mu \gamma_{1}}{\delta_{1}-\gamma_{1}}\right)^{2}+4 a \beta\left(N_{0}+\frac{c \mu \gamma \gamma_{1}}{\delta_{1}-\gamma_{1}}\right)\right]}, \\
N_{2 *}=\frac{\mu \gamma_{1}}{\delta_{1}-\gamma_{1}}, \quad N_{3 *}=\frac{\mu \delta_{1}}{\delta\left(\delta_{1}-\gamma_{1}\right)}\left(\frac{\alpha_{1} N_{1 *}}{\beta+N_{1 *}}-\gamma\right) .
\end{gathered}
$$


Now from the expressions of the above equilibrium points we see that the axial equilibrium $E_{1}$ always exists. The boundary equilibrium $E_{2}$ will exist if the following restrictions on the parameters are satisfied:

$$
\frac{\alpha}{c}>\alpha_{1}>\gamma, \quad N_{0}>\frac{a \beta \gamma}{\alpha_{1}-\gamma}=N_{01}
$$

that is, if the growth rate of detritivores due to consumption of detritus biomass must exceed some threshold value and the constant supply of detritus is higher than some critical value $N_{01}$. The existence of interior equilibrium $E_{*}$ demands

$$
\delta_{1}>\gamma_{1}, \quad N_{1 *}>\frac{\beta \gamma}{\alpha_{1}-\gamma} \quad \text { with } \alpha_{1}>\gamma .
$$

Using the expression for $N_{1 *}$ from (3.2) we ultimately arrive at

$$
N_{0}>\frac{a \beta \gamma}{\alpha_{1}-\gamma}+\frac{\mu \gamma \gamma_{1}}{\delta_{1}-\gamma_{1}}\left(\frac{\alpha}{\alpha_{1}}-c\right)=N_{02},
$$

that is, the uptake rate on detritivores exceeds the mortality rate of the predator of detritivores and the constant supply of detritus exceeds some critical value $N_{02}$.

Before going to obtain the conditions for local stability of various equilibria we first give the criteria for which the detritivores and the predator of detritivores or both of them may become extinct.

Theorem 3.1. Let the inequality

$$
\alpha_{1}<\gamma
$$

hold. Then $\lim _{t \rightarrow \infty} N_{2}(t)=0$.

Proof. From the system (2.5), we have

$$
\begin{aligned}
\frac{d N_{2}}{d t} & =\alpha_{1} \frac{N_{1} N_{2}}{\beta+N_{1}}-\gamma N_{2}-\delta \frac{N_{2} N_{3}}{\mu+N_{2}}=N_{2}\left[\alpha_{1} \frac{N_{1}}{\beta+N_{1}}-\gamma-\delta \frac{N_{3}}{\mu+N_{2}}\right] \\
& \leq N_{2}\left[\alpha_{1} \frac{N_{1}}{\beta+N_{1}}-\gamma\right] \leq N_{2}\left[\alpha_{1}-\gamma\right] \leq 0 \quad \text { by (3.6). }
\end{aligned}
$$

This shows that Theorem 3.1 follows.

Theorem 3.1 shows that if the maximum nutrient uptake rate is less than the mortality rate of the predator, then the predator (detritivores) population is eliminated.

Theorem 3.2. Let the inequality

$$
\delta_{1}<\gamma_{1}
$$

hold. Then $\lim _{t \rightarrow \infty} N_{3}(t)=0$. 
6 Effect of time delay on a detritus-based ecosystem

Proof. From the system (2.5), we have

$$
\begin{aligned}
\frac{d N_{3}}{d t} & =\delta_{1} \frac{N_{2} N_{3}}{\mu+N_{2}}-\gamma_{1} N_{3}=N_{3}\left[\delta_{1} \frac{N_{2}}{\mu+N_{2}}-\gamma_{1}\right] \\
& \leq N_{3}\left[\delta_{1}-\gamma_{1}\right]<0 \quad \text { by }(3.8) .
\end{aligned}
$$

This shows that Theorem 3.2 follows.

This shows that if the maximum uptake rate of detritivores is less than the mortality rate of the predators of detritivores population, then the predators of detritivores population become extinct.

If the conditions stated in Theorems 3.1 and 3.2 are satisfied, the system (2.5) cannot be feasible for persistence and also there will be no equilibrium points for the model under consideration except the axial equilibrium point.

We now study the boundedness of the system.

Theorem 3.3. All the solutions of the model system (2.5) with initial conditions are uniformly bounded.

Proof. The right-hand sides of the system (2.5) are smooth functions of variables $\left(N_{1}, N_{2}\right.$, $N_{3}$ ) and all parameters are nonnegative. Let us consider the time-dependent function

$$
W(t)=N_{1}+\frac{\alpha}{\alpha_{1}} N_{2}+\frac{\alpha \delta}{\alpha_{1} \delta_{1}} N_{3}
$$

Clearly,

$$
\frac{d W(t)}{d t}=\frac{d N_{1}}{d t}+\frac{\alpha}{\alpha_{1}} \frac{d N_{2}}{d t}+\frac{\alpha \delta}{\alpha_{1} \delta_{1}} \frac{d N_{3}}{d t}
$$

Using (2.5) in the above expression we obtain

$$
\begin{aligned}
\frac{d W(t)}{d t}= & {\left[N_{0}-a N_{1}-\alpha \frac{N_{1} N_{2}}{\beta+N_{1}}+c \gamma N_{2}\right]+\frac{\alpha}{\alpha_{1}}\left[\alpha_{1} \frac{N_{1} N_{2}}{\beta+N_{1}}-\gamma N_{2}-\delta \frac{N_{2} N_{3}}{\mu+N_{2}}\right] } \\
& +\frac{\alpha \delta}{\alpha_{1} \delta_{1}}\left[\delta_{1} \frac{N_{2} N_{3}}{\mu+N_{2}}-\gamma_{1} N_{3}\right]=N_{0}-a N_{1}-\gamma\left(\frac{\alpha}{\alpha_{1}}-c\right) N_{2}-\frac{\alpha \delta \gamma_{1}}{\alpha_{1} \delta_{1}} N_{3} \\
\leq & N_{0}-\omega W(t),
\end{aligned}
$$

where $\omega$ is chosen as the minimum of $\left\{a, \gamma\left(1-c\left(\alpha_{1} / \alpha\right)\right), \gamma_{1}\right\}$. Thus

$$
\frac{d W(t)}{d t}+\omega W(t) \leq N_{0}
$$

Now applying the theorem of differential inequalities [4], we obtain

$$
0<W(t) \leq W(0) e^{-\omega t}+\frac{N_{0}}{\omega} .
$$


When the time $t \rightarrow \infty$,

$$
0 \leq W \leq \frac{N_{0}}{\omega}
$$

Hence all the solutions of the system (2.5) that initiate in $\left\{\mathbb{R}_{+}^{3}-0\right\}$ are bounded.

\section{Local stability analysis of deterministic model}

In this section we consider the stability behaviour of the model system for the system of (2.5). In the previous section we saw that $E_{1}\left(N_{0} / a, 0,0\right), E_{2}\left(N_{1 b}, N_{2 b}, 0\right)$, and $E_{*}\left(N_{1 *}, N_{2 *}\right.$, $N_{3 *}$ ) are the exhaustive list of feasible equilibrium points if and only if the conditions (3.3)-(3.5) on the parameters are satisfied. Now we study the stability of the model system around different equilibrium points.

At the axial equilibrium point $E_{1}$, the Jacobian matrix is given by

$$
J_{E_{1}}=\left(\begin{array}{ccc}
-a & -\left(\frac{\alpha N_{0}}{a \beta+N_{0}}-c \gamma\right) & 0 \\
0 & \frac{\alpha_{1} N_{0}}{a \beta+N_{0}}-\gamma & 0 \\
0 & 0 & -\gamma_{1}
\end{array}\right) .
$$

The characteristic equation corresponding to $J_{E_{1}}$ is

$$
(-a-\lambda)\left(\frac{\alpha_{1} N_{0}}{a \beta+N_{0}}-\gamma-\lambda\right)\left(-\gamma_{1}-\lambda\right)=0
$$

The axial equilibrium point $E_{1}$ will be locally asymptotically stable if all the eigenvalues are negative $[4,9,27]$. Here the eigenvalues are

$$
\lambda_{1}=-a, \quad \lambda_{2}=\frac{\alpha_{1} N_{0}}{a \beta+N_{0}}-\gamma, \quad \lambda_{3}=-\gamma_{1} .
$$

Thus if

$$
\frac{\alpha_{1} N_{0}}{a \beta+N_{0}}-\gamma<0, \quad \text { that is, if } N_{0}<\frac{a \beta \gamma}{\alpha_{1}-\gamma}=N_{01},
$$

then point $E_{1}$ is locally asymptotically stable. Therefore, if the constant supply of the detritus is less than some critical value $N_{01}$, then the axial equilibrium point is locally asymptotically stable.

Let us now study the boundary equilibrium point $E_{2} \equiv\left(N_{1 b}, N_{2 b}, 0\right)$. The Jacobian matrix at $E_{2}$ is given by

$$
J_{E_{2}}=\left(\begin{array}{ccc}
f_{1}\left(N_{0}\right) & -\gamma\left(\frac{\alpha}{\alpha_{1}}-c\right) & 0 \\
f_{2}\left(N_{0}\right) & 0 & f_{3}\left(N_{0}\right) \\
0 & 0 & f_{4}\left(N_{0}\right)
\end{array}\right) \text {, }
$$


where

$$
\begin{gathered}
f_{1}\left(N_{0}\right)=-a-\frac{\alpha}{\beta \gamma} \frac{\left(1-\gamma / \alpha_{1}\right)^{2}}{\alpha / \alpha_{1}-c}\left(N_{0}-\frac{a \beta \gamma}{\alpha_{1}-\gamma}\right), \quad f_{2}\left(N_{0}\right)=\frac{\left(1-\gamma / \alpha_{1}\right)^{2}}{\alpha / \alpha_{1}-c}\left(N_{0}-\frac{a \beta \gamma}{\alpha_{1}-\gamma}\right), \\
f_{3}\left(N_{0}\right)=\frac{-\delta\left(N_{0}-a \beta \gamma /\left(\alpha_{1}-\gamma\right)\right)}{\mu \gamma\left(\alpha / \alpha_{1}-c\right)+\left(N_{0}-a \beta \gamma /\left(\alpha_{1}-\gamma\right)\right)}, \\
f_{4}\left(N_{0}\right)=\frac{-\mu \gamma \gamma_{1}\left(\alpha / \alpha_{1}-c\right)+\left(\delta_{1}-\gamma_{1}\right)\left(N_{0}-a \beta \gamma /\left(\alpha_{1}-\gamma\right)\right)}{\mu \gamma\left(\alpha / \alpha_{1}-c\right)+\left(N_{0}-a \beta \gamma /\left(\alpha_{1}-\gamma\right)\right)} .
\end{gathered}
$$

From the community matrix $J_{E_{2}}$ it is clear that the eigenvalues of $J_{E_{2}}$ are $\lambda_{3}=f_{4}\left(N_{0}\right)$ and the eigenvalues of the matrix

$$
J_{E_{2}^{\prime}}=\left(\begin{array}{cc}
f_{1}\left(N_{0}\right) & -\gamma\left(\frac{\alpha}{\alpha_{1}}-c\right) \\
f_{2}\left(N_{0}\right) & 0
\end{array}\right)
$$

Now $\lambda_{3}$ is negative if

$$
f_{4}\left(N_{0}\right)<0 \Longrightarrow N_{0}<\frac{a \beta \gamma}{\alpha_{1}-\gamma}+\frac{\mu \gamma \gamma_{1}}{\delta_{1}-\gamma_{1}}\left(\frac{\alpha}{\alpha_{1}}-c\right)=N_{02}
$$

Since $\operatorname{Tr}\left(J_{E_{2}^{\prime}}\right)=f_{1}\left(N_{0}\right)<0$ if $N_{0}>N_{01}$ and $\operatorname{det}\left(J_{E_{2}^{\prime}}\right)=\gamma\left(\alpha / \alpha_{1}-c\right) f_{2}\left(N_{0}\right)>0$, the other two eigenvalues are negative. Thus we conclude that the boundary equilibrium point $E_{2}$ is exponentially asymptotically stable if

$$
N_{01}<N_{0}<N_{02}
$$

Thus, the boundary steady state will be locally asymptotically stable if the constant supply of detritus belongs to two critical values $N_{01}$ and $N_{02}$.

Now we study the stability of the most interesting interior equilibrium point $E_{*}$ which is also known as coexisting equilibrium point. As we are interested of the stability of the steady state $E_{*}$ which is singular point in the phase plane of (2.5), a linear stability analysis around the equilibrium point $E_{*}$ is equivalent to the phase space analysis. We linearize the system about the interior equilibrium point $E_{*}$ for which the community matrix $J_{E_{*}}$ is as follows:

$$
J_{E_{*}}=\left(\begin{array}{ccc}
a_{11} & a_{12} & 0 \\
a_{21} & a_{22} & a_{23} \\
0 & a_{32} & 0
\end{array}\right)
$$


where

$$
\begin{gathered}
a_{11}=-a-\frac{\alpha \beta \mu \gamma_{1}}{\delta_{1}-\gamma_{1}} \frac{1}{\left(\beta+N_{1 *}\right)^{2}}, \quad a_{12}=-\frac{\delta_{1}-\gamma_{1}}{\mu \gamma_{1}}\left(N_{0}-a N_{1 *}\right), \\
a_{21}=\frac{\alpha_{1} \beta \mu \gamma_{1}}{\delta_{1}-\gamma_{1}} \frac{1}{\left(\beta+N_{1 *}\right)^{2}}, \quad a_{22}=\frac{\alpha_{1} \gamma_{1}}{\alpha \delta_{1}}\left[\frac{\delta_{1}-\gamma_{1}}{\mu \gamma_{1}}\left(N_{0}-a N_{1 *}\right)+\gamma\left(\frac{\alpha}{\alpha_{1}}-c\right)\right], \\
a_{23}=-\frac{\delta \gamma_{1}}{\delta_{1}}, \quad a_{32}=\frac{\alpha_{1}\left(\delta_{1}-\gamma_{1}\right)}{\alpha \delta}\left[\frac{\delta_{1}-\gamma_{1}}{\mu \gamma_{1}}\left(N_{0}-a N_{1 *}\right)+\gamma\left(\frac{\alpha}{\alpha_{1}}-c\right)\right]
\end{gathered}
$$

with the restrictions (3.3), (3.4), and (3.5). From the above community matrix, we obtain the characteristic equation

$$
\lambda^{3}+p \lambda^{2}+q \lambda+r=0
$$

where

$$
p=-\left(a_{11}+a_{22}\right), \quad q=a_{11} a_{22}-a_{12} a_{21}-a_{23} a_{32}, \quad r=a_{11} a_{23} a_{32} .
$$

The characteristic equation (4.12) will have roots with negative real part if it satisfies the Routh-Hurwitz criteria [27, 28], that is, $p, r$, and $p q-r$ are all positive.

To find the conditions for which $p, r$, and $p q-r$ are positive we use the bound of $N_{1 *}$ as

$$
-\frac{c \mu \gamma \gamma_{1}}{\delta_{1}-\gamma_{1}}<N_{0}-a N_{1 *}<a \beta+\frac{\mu \gamma_{1}}{\delta_{1}-\gamma_{1}}(\alpha-c \gamma)
$$

Now $r$ is positive if

$$
N_{0}<\frac{a c \beta \gamma}{\alpha-c \gamma}=N_{03}
$$

$p$ will be positive if $a_{11}+a_{22}<0$, that is, if

$$
N_{0}<\sqrt{\frac{b}{d-a}}+e=N_{04} \quad \text { along with } \alpha_{1}<\frac{\alpha}{2 c}\left(\frac{a \delta_{1}}{\gamma \gamma_{1}}-1\right),
$$

where $b=a^{2} \alpha \beta\left(\mu \gamma_{1} /\left(\delta_{1}-\gamma_{1}\right)\right), d=\left(\alpha_{1} \gamma \gamma_{1} / \alpha \delta_{1}\right)\left(\alpha / \alpha_{1}-2 c\right), e=\left(\mu \gamma_{1} /\left(\delta_{1}-\gamma_{1}\right)\right)(\alpha-c \gamma)$. Now $p q-r>0$ holds if

$$
\left(a_{11}+a_{22}\right)\left(a_{12} a_{21}-a_{11} a_{22}\right)+a_{22} a_{23} a_{32}>0 .
$$

Using the bound of $N_{1 *}$ from (4.14) one can obtain the following result:

$$
N_{0}>\frac{f g}{f+g}\left(1+\frac{g h}{f+h}\right)-\frac{c \mu \gamma \gamma_{1}}{\delta_{1}-\gamma_{1}}=N_{05},
$$

where $f=\left(\alpha_{1} \gamma_{1} / \alpha \delta_{1}\right)\left(c \mu \gamma \gamma_{1} /\left(\delta_{1}-\gamma_{1}\right)\right), g=a \beta+\left(\alpha \mu \gamma_{1} /\left(\delta_{1}-\gamma_{1}\right)\right), h=a \beta+d$. 
Let $\hat{N}_{0}=\max \left\{N_{02}, N_{05}\right\}$ and $\tilde{N}_{0}=\min \left\{N_{03}, N_{04}\right\}$, then from (3.5) and (4.8), we see that if

$$
\widehat{N}_{0}<N_{0}<\tilde{N}_{0}
$$

then the interior equilibrium point $E_{*}$ is locally asymptotically stable. Therefore, the interior steady state $E_{*}$ is locally asymptotically stable if the constant supply of detritus belongs to the two critical values $\hat{N}_{0}$ and $\tilde{N}_{0}$.

Now we show that the system (2.5) is persistent.

Theorem 4.1. If the inequality

$$
N_{0}>\frac{a \beta \gamma}{\alpha_{1}-\gamma}+\frac{\mu \gamma \gamma_{1}}{\delta_{1}-\gamma_{1}}\left(\frac{\alpha}{\alpha_{1}}-c\right), \quad \delta_{1} \frac{N_{2 b}}{\mu+N_{2 b}}-\gamma_{1}>0,
$$

then the system is persistent.

Proof. In the above discussion we saw that the equilibrium on the prey axis is stable when $N_{0}<a \beta \gamma /\left(\alpha_{1}-\gamma\right)$. If $N_{0}>a \beta \gamma /\left(\alpha_{1}-\gamma\right)$, then the point on the prey axis becomes unstable saddle point. This instability condition of the system in (2.5) at the axial steady state leads to the stability of the boundary equilibrium point $E_{2}$ in the $N_{1} N_{2}$-plane.

Again, if

$$
N_{0}>\frac{a \beta \gamma}{\alpha_{1}-\gamma}+\frac{\mu \gamma \gamma_{1}}{\delta_{1}-\gamma_{1}}\left(\frac{\alpha}{\alpha_{1}}-c\right),
$$

then the boundary steady state becomes unstable. The instability condition of the boundary equilibrium point leads to the stability of the interior equilibrium point within the positive octant of $\mathrm{N}_{1} \mathrm{~N}_{2} \mathrm{~N}_{3}$-space.

Now, let us suppose that

$$
H\left(N_{1}, N_{2}, N_{3}\right)=\delta_{1} \frac{N_{2}}{\mu+N_{2}}-\gamma_{1}
$$

Then

$$
H\left(N_{1 b}, N_{2 b}, 0\right)=\delta_{1} \frac{N_{2 b}}{\mu+N_{2 b}}-\gamma_{1}>0 \quad \text { by }(4.20) .
$$

Therefore, according to Theorem 5.1 of Freedman and Waltman [12], the model system (2.5) is persistent.

\section{Delay model: local stability analysis}

In this section we consider the discrete time delay model which is in a modified version of deterministic model system (2.5). Construction of discrete time delay model is based upon the following assumptions: (i) the conversion from dead biomass of detritivores to detritus through decomposition is not an instantaneous phenomenon, it takes some time $\tau_{1}$, (ii) on the other hand, the consumed biomass of detritus by the detritivores contribute 
to the biomass of detritivores after a finite-time interval $\tau_{2}$, which is known as gestation delay, (iii) $\tau_{3}$ is the time required for conversion of detritivores biomass to predator of detritivore's biomass.

So, we introduce the discrete time delay $\tau_{1}$ in the recycling term of $N_{2}$ in the growth equation of $N_{1}$ population and obtain the growth equation of detritus as

$$
\frac{d N_{1}}{d t}=N_{0}-a N_{1}-\frac{\alpha N_{1} N_{2}}{\beta+N_{1}}+c \gamma N_{2}\left(t-\tau_{1}\right)
$$

We introduce the gestation delay $\tau_{2}$ in the functional response term of detritus in the growth equation of detritivores as follows:

$$
\frac{d N_{2}}{d t}=\alpha_{1} \frac{N_{1}\left(t-\tau_{2}\right) N_{2}}{\beta+N_{1}\left(t-\tau_{2}\right)}-\gamma N_{2}-\delta \frac{N_{2} N_{3}}{\mu+N_{2}} .
$$

Finally, we introduce the gestation delay $\tau_{3}$ in the functional response term involved with the growth equation of predators of detritivores to obtain the following:

$$
\frac{d N_{3}}{d t}=\delta_{1} \frac{N_{2}\left(t-\tau_{3}\right) N_{3}}{\mu+N_{2}\left(t-\tau_{3}\right)}-\gamma_{1} N_{3}
$$

Thus we have a delay differential equation model system (5.1a)-(5.1c) for the detritusbased food chain model where the discrete time delays $\tau_{1}, \tau_{2}, \tau_{3}$ are all positive constants. The initial conditions for the model system (5.1) are given by

$$
\begin{array}{cl}
N_{1}(t)=N_{1}^{0}(t) \quad \text { for }-\tau_{2} \leq t \leq 0, & N_{2}(t)=N_{2}^{0}(t) \quad \text { for }-\tau_{4} \leq t \leq 0, \\
\tau_{4}=\max \left\{\tau_{1}, \tau_{3}\right\}, & N_{3}(0)=N_{30}>0
\end{array}
$$

where $N_{i}^{0}(t) \in C\left(\left[-\tau_{i}, 0\right], \mathbb{R}_{+}\right), i=2,4$, are given nonnegative functions.

The steady states of the delayed system (5.1) are same as those for the system (2.5). Thus the steady states of the system (5.1) are $E_{1}\left(N_{0} / a, 0,0\right), E_{2}\left(N_{1 b}, N_{2 b}, 0\right)$, and $E_{*}\left(N_{1 *}\right.$, $\left.N_{2 *}, N_{3 *}\right)$, where expressions for $N_{1 *}, N_{2 *}$, and $N_{3 *}$ are given in (3.2).

Let us now linearize the above system of nonlinear delay differential equations (5.1) about the interior equilibrium point $E_{*}\left(N_{1 *}, N_{2 *}, N_{3 *}\right)$. Let $x \equiv x(t), y \equiv y(t), z \equiv z(t)$ be the small perturbations from the equilibrium values $N_{i *}, i=1,2,3$, substituting $N_{1}=$ $N_{1 *}+x, N_{2}=N_{2 *}+y, N_{3}=N_{3 *}+z$ in (5.1) and retaining linear terms we get the following system of linear ordinary differential equations [20]:

$$
\frac{d x}{d t}=a_{11} x+a_{12} y\left(t-\tau_{1}\right), \quad \frac{d y}{d t}=a_{21} x\left(t-\tau_{2}\right)+a_{22} y+a_{23} z, \quad \frac{d z}{d t}=a_{32} y\left(t-\tau_{3}\right),
$$

where the coefficients are given in (4.11). The characteristic equation corresponding to the linearized system (5.2) is given by [15]

$$
\Delta\left(\lambda, \tau_{1}, \tau_{2}, \tau_{3}\right) \equiv \lambda^{3}+p \lambda^{2}+\left[\theta+\rho e^{-\lambda\left(\tau_{1}+\tau_{2}\right)}+\phi e^{-\lambda \tau_{3}}\right] \lambda+r e^{-\lambda \tau_{3}}=0,
$$


where $p, \theta, \rho, \phi, r$ are given by

$p=-\left(a_{11}+a_{22}\right), \quad \theta=a_{11} a_{22}, \quad \rho=-a_{12} a_{21}, \quad \phi=-a_{23} a_{32}, \quad r=a_{11} a_{23} a_{32}$.

Let us now substitute $T_{1}=\tau_{1}+\tau_{2}, T_{2}=\tau_{3}$ in (5.3) in order to simplify the forthcoming mathematical calculations,

$$
\Delta\left(\lambda, T_{1}, T_{2}\right) \equiv \lambda^{3}+p \lambda^{2}+\left[\theta+\rho e^{-\lambda T_{1}}+\phi e^{-\lambda T_{2}}\right] \lambda+r e^{-\lambda T_{2}}=0 .
$$

Let us try to find the condition for nonexistence of delay-induced instability by using the following theorem [15].

Theorem 5.1. A set of necessary and sufficient conditions for $E_{*}$ is to be locally asymptotically stable in presence of time delays, $T_{j}, j=1,2$, if the following conditions are satisfied:

(i) the real parts of all the roots of $\Delta(\lambda, 0,0)=0$ are negative;

(ii) for all real s and any $T_{j}>0$, the following holds:

$$
\Delta\left(\text { is, } T_{1}, T_{2}\right) \neq 0, \quad \text { where } i=\sqrt{-1} .
$$

In (5.5), substituting $T_{1}=0=T_{2}$ we get

$$
\Delta(\lambda, 0,0)=\lambda^{3}+p \lambda^{2}+q \lambda+r=0
$$

which is same as (4.12). The real parts of the roots of (5.5) are negative if (4.18) holds along with the existence conditions for $E_{*}$. Hence the first condition of Theorem 5.1 is satisfied.

Let us suppose there exists a real $s>0$ for some $T_{j} \geq 0, j=1,2$, such that $\Delta\left(i s, T_{1}, T_{2}\right)=0$, then the characteristic equation (5.5) will have a pair of purely imaginary roots and hence $E_{*}$ is not asymptotically stable in presence of discrete time delay.

For $s=0, \Delta\left(0, T_{1}, T_{2}\right)=r>0$, that is, $\Delta\left(0, T_{1}, T_{2}\right) \neq 0$.

Next we assume $s \neq 0$,

$$
\Delta\left(i s, T_{1}, T_{2}\right)=-i s^{3}-p s^{2}+\left[\theta+\rho e^{-i s T_{1}}+\phi e^{-i s T_{2}}\right] i s+r e^{-i s T_{2}} .
$$

Separating real and imaginary parts of the equation $\Delta\left(i s, T_{1}, T_{2}\right)=0$, we get

$$
\begin{gathered}
p s^{2}=s \rho \sin s T_{1}+s \phi \sin s T_{2}+r \cos s T_{2}, \\
s^{3}-s \theta=s \rho \cos s T_{1}+s \phi \cos s T_{2}-r \sin s T_{2} .
\end{gathered}
$$

Squaring the above two equations and then addition leads to a sixth degree polynomial equation in " $s$ " as follows:

$$
p^{2} s^{4}+s^{2}\left(s^{2}-\theta\right)^{2}=\rho^{2} s^{2}+\phi^{2} s^{2}+r^{2}+2 \rho \phi s^{2} \cos s\left(T_{1}-T_{2}\right)+2 r s \rho \sin s\left(T_{1}-T_{2}\right) .
$$




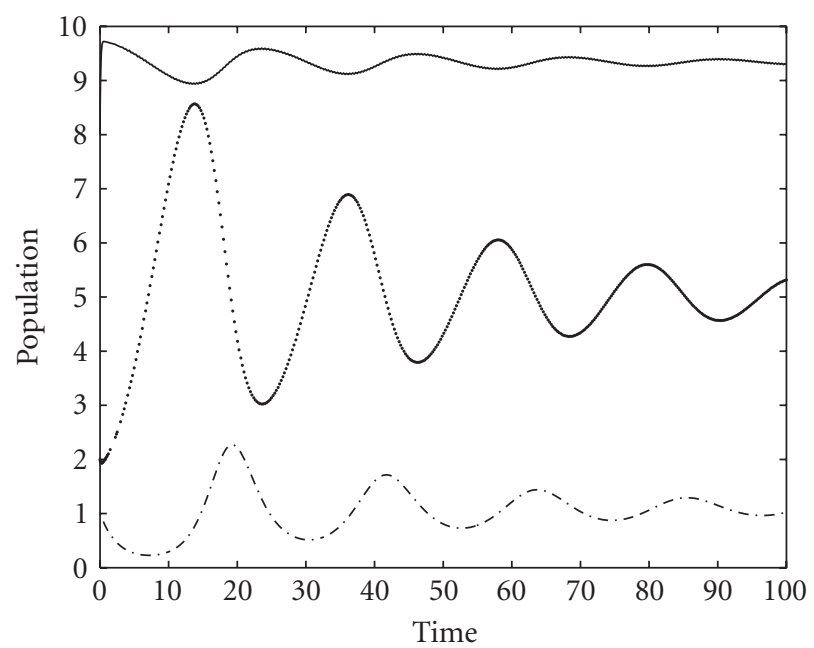

— Detritus

.... Detritivores

.... Predator of detritivores

Figure 5.1. Stable population distribution of the model (5.1) with the parametric values as described in the text.

Let us denote the right-hand side of (5.10) by $f(s)$. Using the trigonometrical inequalities $0 \leq|\sin \Upsilon| \leq 1$ and $0 \leq|\cos \Upsilon| \leq 1$ to $f(s)$, we arrive at

$$
f(s) \leq\left(\rho^{2}+\phi^{2}\right) s^{2}+r^{2}+2 \rho \phi s^{2}+2 \rho r|s| .
$$

Thus, (5.10) can be put in the following inequality:

$$
p^{2} s^{4}+\left(s^{2}-\theta\right)^{2} s^{2} \leq\left(\rho^{2}+\phi^{2}\right) s^{2}+2 \rho \phi s^{2}+2 \rho r|s|+r^{2} .
$$

Therefore, the sufficient conditions for the nonexistence of a real number " $s$ " satisfying $\Delta\left(i s, T_{1}, T_{2}\right)=0$ depend upon the satisfaction of the inequality

$$
s^{6}+\left(p^{2}-2 \theta\right) s^{4}+\left[\theta^{2}-(\rho+\phi)^{2}\right] s^{2}-2 \rho r|s|-r^{2}>0 .
$$

It is quite difficult to find out the condition for which the inequality in (5.12) will be satisfied for all real $s$ and hence the stability condition independent of length of discrete time delay cannot be obtained for the present model system. Thus, the second condition of Theorem 5.1 is never satisfied for the model system under consideration. We illustrate this result with help of numerical simulation.

For numerical simulation we take a hypothetical set of parameter values as $N_{0}=100$, $a=10, \alpha=10, \beta=25, \alpha_{1}=8, \gamma=2, \delta=4, \mu=20, \delta_{1}=3, \gamma_{1}=0.6$, and $c=0.7$. For $\tau_{1}=$ $0.5, \tau_{2}=0.1$, and $\tau_{3}=0.2$, we get stable population distribution and the solution trajectory approaches $E_{*}(9.34,5,1.099)$ in phase space (see Figures 5.1 and 5.2). If we increase 


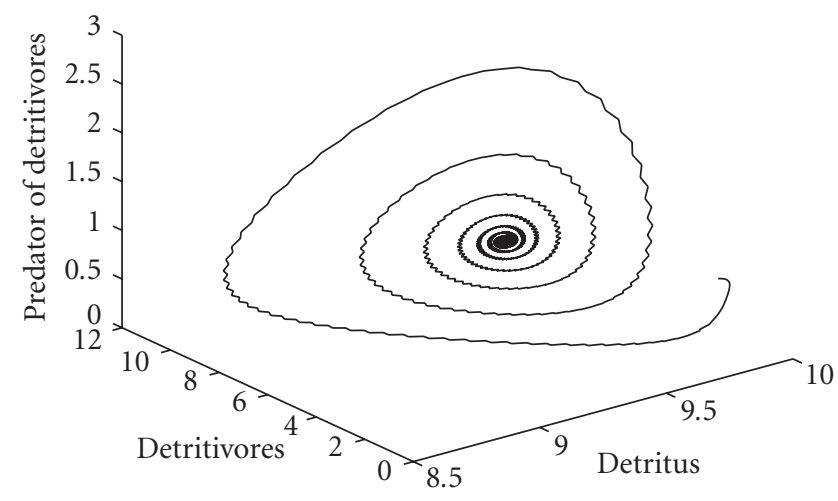

Figure 5.2. Stable phase-space diagram corresponding to Figure 5.1.

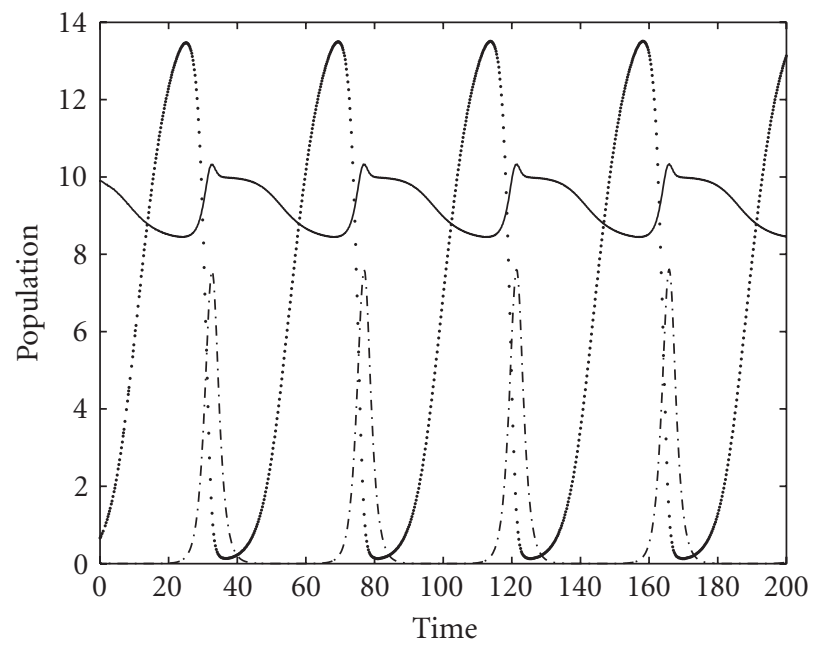

- Detritus

.... Detritivores

.... Predator of detritivores

Figure 5.3. Unstable population distribution of the model (5.1) with the parametric values as described in the text.

the magnitude of delay parameters from $\tau_{1}=0.5, \tau_{2}=0.1$, and $\tau_{3}=0.2$ to $\tau_{1}=3.5$, $\tau_{2}=0.3$, and $\tau_{3}=1.5$ (keeping all other parameters fixed), then the system loses its stability and we obtain unstable solution (see Figure 5.3).

Hence we can conclude that the system is not always stable for arbitrary values of delay parameters and length of discrete time delay plays a vital role behind the stability behaviour of the model system. In the next section we try to find the upper bounds of delay parameters for preservation of local asymptotic stability of $E_{*}$. 


\section{Estimation for the length of delay to preserve stability}

In this section we try to find an estimate for the length of time delay for the system (5.1) which will preserve the stability behaviour of $E_{*}$. For this purpose we assume that in absence of delay, the interior equilibrium point $E_{*}$ is locally asymptotically stable, (4.19) gives the condition for local asymptotic stability of $E_{*}$ when $T_{1}=0=T_{2}$. By continuity and sufficiently small $T_{1}, T_{2}>0$ all eigenvalues of (5.5) will have negative real parts, provided one can guarantee that no eigenvalue with positive real part bifurcates from infinity (which could happen for retarded system sometimes) [32]. We obtain the length of delay which will preserve stability of $E_{*}$, by applying Nyquist criteria [33]. For this purpose we consider the system of (5.1) and assume that the variables involved with the system of equations are continuous functions defined over $[T, \infty)$, where $T=\min \left\{-T_{1},-T_{2}\right\}$ and satisfying initial conditions (5.1d). We recall the linearized system of (5.2) corresponding to the model system (5.1) as follows:

$$
\frac{d x}{d t}=a_{11} x+a_{12} y\left(t-\tau_{1}\right), \quad \frac{d y}{d t}=a_{21} x\left(t-\tau_{2}\right)+a_{22} y+a_{23} z, \quad \frac{d z}{d t}=a_{32} y\left(t-\tau_{3}\right),
$$

where $a_{i j}$ 's are same as in (4.11). Let $\bar{x}=\bar{x}(s), \bar{y}=\bar{y}(s)$, and $\bar{z}=\bar{z}(s)$ denote the Laplace transform of $x, y$, and $z$, respectively. Taking Laplace transform of the system of (6.1), we get

$$
\begin{gathered}
\left(s-a_{11}\right) \bar{x}=a_{12}\left[\bar{y}+k_{1}(s)\right] e^{-s \tau_{1}}+x(0), \quad\left(s-a_{22}\right) \bar{y}=a_{21}\left[\bar{x}+k_{2}(s)\right] e^{-s \tau_{2}}+a_{23} \bar{z}+y(0), \\
s \bar{z}(s)=a_{32}\left[\bar{y}(s)+k_{3}(s)\right] e^{-s \tau_{3}}+z(0),
\end{gathered}
$$

where

$$
k_{1}(s)=\int_{-\tau_{1}}^{0} y(t) e^{-s t} d t, \quad k_{2}(s)=\int_{-\tau_{2}}^{0} x(t) e^{-s t} d t, \quad k_{3}(s)=\int_{-\tau_{3}}^{0} y(t) e^{-s t} d t
$$

Solving the above system of equations for $\bar{z}(s)$ we obtain

$$
\bar{z}(s)=\frac{\Gamma\left(s, \tau_{1}, \tau_{2}, \tau_{3}, a_{j k}\right)}{s^{3}+p s^{2}+\left[\theta+\rho e^{-s\left(\tau_{1}+\tau_{2}\right)}+\phi e^{-s \tau_{3}}\right] s+r e^{-s \tau_{3}}},
$$

where $\Gamma(\cdot)$ is function of $s, \tau_{i}, i=1,2,3$, and $a_{j k}$ 's are given in (4.11). Let

$$
G(s)=s^{3}+p s^{2}+\left[\theta+\rho e^{-s\left(\tau_{1}+\tau_{2}\right)}+\phi e^{-s \tau_{3}}\right] s+r e^{-s \tau_{3}}
$$

then $G(s)$ is the polynomial involved with the characteristic equation (5.5).

The inverse Laplace transformation of $\bar{z}(s)$ will have terms which increase exponentially with time if $\bar{z}(s)$ has poles with positive real part. Thus for local asymptotic stability of interior equilibrium point $E_{*}$, it is necessary and sufficient that all poles of $\bar{z}(s)$ have negative real parts. For this purpose we apply Nyquist criteria to ensure that $\bar{z}(s)$ has any 
pole in the right-half of complex $s$-plane. Nyquist criteria lead us to the following conditions (for details see [33]):

$$
\operatorname{Im} G\left(i w_{0}\right)>0, \quad \operatorname{Re} G\left(i w_{0}\right)=0,
$$

where $w_{0}$ is the smallest positive root of the equation $\operatorname{Re} G(i w)=0$. In (6.4), putting $s=i \omega$ we obtain

$$
\begin{aligned}
G(i w)= & -i w^{3}-p w^{2}+\left[\theta+\rho\left(\cos w T_{1}-i \sin w T_{1}\right)+\phi\left(\cos w T_{2}-i \sin w T_{2}\right)\right] i w \\
& +r\left(\cos w T_{2}-i \sin w T_{2}\right) .
\end{aligned}
$$

Therefore, the conditions (6.5) can be written as

$$
\begin{gathered}
-w_{0}^{3}+\theta w_{0}>-\rho w_{0} \cos w_{0} T_{1}-\phi w_{0} \cos w_{0} T_{2}+r \sin w_{0} T_{2} \\
-p w_{0}^{2}=-\rho w_{0} \sin w_{0} T_{1}-\phi w_{0} \sin w_{0} T_{2}-r \cos w_{0} T_{2} .
\end{gathered}
$$

To get an estimate for the length of delay which preserves the stability we utilize the following conditions:

$$
\begin{gathered}
-w^{3}+\theta w>-\rho w \cos w T_{1}-\phi w \cos w T_{2}+r \sin w T_{2} \\
p w^{2}=\rho w \sin w T_{1}+\phi w \sin w T_{2}+r \cos w T_{2}
\end{gathered}
$$

Therefore, $E_{*}$ will be stable if the inequality (6.8) holds for $w=w_{0}$, where $w_{0}$ is the smallest positive root of (6.9). Our target is to find an upper bound $w_{+}$of $w$ which would be independent of $T_{1}$ and $T_{2}$ such that (6.8) holds for all values of $w, 0 \leq w \leq w_{+}$, and hence, in particular, for $w=w_{0}$.

Maximizing the expression in the right-hand side of (6.9) subject to the restriction $|\cos \xi| \leq 1$ and $|\sin \xi| \leq 1$ we obtain from (6.9)

$$
p w^{2} \leq(\rho+\phi) w+r
$$

Thus the unique positive solution of

$$
p w^{2}-(\rho+\phi) w-r=0
$$

denoted by $w_{+}$is always greater than or equal to $w_{0}$. Hence, if

$$
w_{+}=\frac{1}{2 p}\left[\rho+\phi+\sqrt{(\rho+\phi)^{2}+4 p r}\right]
$$

then from (6.9) we see that $w_{0}<w_{+}$. Here $w_{+}$is independent of $T_{1}$ and $T_{2}$. Now we need to find an estimate on $T_{1}$ and $T_{2}$ so that (6.8) holds for $0 \leq w \leq w_{+}$. Dividing (6.8) by $w$, we get

$$
w^{2}<\theta+\rho \cos w T_{1}+\phi \cos w T_{2}-\frac{r}{w} \sin w T_{2}
$$


Note that at $\tau_{j}=0, j=1,2,3$, that is, at $T_{j}=0, j=1,2,(6.13)$ reduces to

$$
w^{2}<\theta+\rho+\phi
$$

and from (6.9), we obtain

$$
w^{2}=\frac{r}{p}
$$

Therefore, from the conditions (6.14) and (6.15) we obtain $r / p<\theta+\rho+\phi$. Using (5.4), we see that $p q-r>0$ which is already satisfied under Routh-Hurwitz criteria. This justifies the validity of inequality (6.13) for $T_{j}=0, j=1,2$, and $w=w_{0}$.

So, by continuity it will hold for small enough $T_{j}>0, j=1,2$, at $w=w_{0}$. Now substituting the expression of $w^{2}$ from (6.9) in (6.13) we obtain

$$
\frac{1}{p}\left[\rho w \sin w T_{1}+\phi w \sin w T_{2}+r \cos w T_{2}\right]<\theta+\rho \cos w T_{1}+\phi \cos w T_{2}-\frac{r}{w} \sin w T_{2}
$$

or, equivalently,

$$
\begin{aligned}
& {\left[\frac{\rho w}{p} \sin w T_{1}+\rho\left(1-\cos w T_{1}\right)\right]+\left[\left|\frac{\phi w}{p}+\frac{r}{w}\right| \sin w T_{2}+\left|\phi-\frac{r}{p}\right|\left(1-\cos w T_{2}\right)\right]} \\
& <\theta+\rho+\phi-\frac{r}{p}=\eta \text { (say). }
\end{aligned}
$$

Let us denote the left-hand side of (6.17) by $\Theta_{1}\left(T_{1}, w\right)+\Theta_{2}\left(T_{2}, w\right)$. Using trigonometric inequalities $\sin w T_{j} \leq w T_{j}$ and $1-\cos w T_{j} \leq(1 / 2) w^{2} T_{j}^{2}$ for $j=1,2$, we obtain the following inequality from (6.17):

$$
\begin{aligned}
\Theta_{1}\left(T_{1}, w\right)+\Theta_{2}\left(T_{2}, w\right) & \leq\left[\frac{\rho w^{2}}{p} T_{1}+\frac{1}{2} \rho w^{2} T_{1}^{2}\right]+\left[\left|\frac{\phi w}{p}+\frac{r}{w}\right| w T_{2}+\frac{1}{2}\left|\phi-\frac{r}{p}\right| w^{2} T_{2}^{2}\right] \\
& \equiv \Phi_{1}\left(T_{1}, w\right)+\Phi_{2}\left(T_{2}, w\right) .
\end{aligned}
$$

We note that for $0 \leq w \leq w_{+}$,

$$
\Theta_{1}\left(T_{1}, w\right)+\Theta_{2}\left(T_{2}, w\right) \leq \Phi_{1}\left(T_{1}, w\right)+\Phi_{2}\left(T_{2}, w\right) \leq \Phi_{1}\left(T_{1}, w_{+}\right)+\Phi_{2}\left(T_{2}, w_{+}\right) .
$$

Now if

$$
\Phi_{1}\left(T_{1}, w_{+}\right)+\Phi_{2}\left(T_{2}, w_{+}\right)<\eta
$$

then from the limit of $w$ we can conclude that

$$
\Theta_{1}\left(T_{1}, w_{0}\right)<\eta, \quad \Theta_{2}\left(T_{2}, w_{0}\right)<\eta .
$$

Let us suppose that

$$
\Phi_{1}\left(T_{1}, w_{+}\right)=F_{1} \eta, \quad \Phi_{2}\left(T_{2}, w_{+}\right)=F_{2} \eta,
$$


where $F_{1}+F_{2}=1$, then

$$
\begin{aligned}
& A_{1} T_{1}^{2}+B_{1} T_{1}-F_{1} \eta=0, \\
& A_{2} T_{2}^{2}+B_{2} T_{2}-F_{2} \eta=0,
\end{aligned}
$$

where

$$
A_{1}=\frac{1}{2} \rho w_{+}^{2}, \quad A_{2}=\frac{1}{2}\left|\phi-\frac{r}{p}\right| w_{+}^{2}, \quad B_{1}=\frac{\rho}{p} w_{+}^{2}, \quad B_{2}=\left|\frac{\phi}{p}+\frac{r}{w_{+}^{2}}\right| w_{+}^{2} .
$$

Let $T_{1}^{+}$and $T_{2}^{+}$be the unique positive roots of (6.23a) and (6.23b), respectively. Then

$$
T_{1}^{+}=\frac{1}{2 A_{1}}\left[-B_{1}+\sqrt{B_{1}^{2}+4 A_{1} F_{1} \eta}\right], \quad T_{2}^{+}=\frac{1}{2 A_{2}}\left[-B_{2}+\sqrt{B_{2}^{2}+4 A_{2} F_{2} \eta}\right] .
$$

As $F_{1}$ and $F_{2}$ are two positive real numbers satisfying $F_{1}+F_{2}=1$, one can find the exact expressions for $T_{1}^{+}$and $T_{2}^{+}$for a particular choice of numerical values for $F_{1}$ and $F_{2}$. Interested readers may concern the work by Das and Roy [7] for a suitable illustration. Thus, for $0<T_{1}<T_{1}^{+}$and $0<T_{2}<T_{2}^{+}$, the Nyquist criteria hold. $T_{1}^{+}$and $T_{2}^{+}$give estimates for the length of delay for which stability is preserved. For the estimate of $T_{1}^{+}, A_{1}$ must be positive. It is ensured by the existence of the interior equilibrium $E_{*}$ when

$$
N_{0}<\frac{a c \beta \gamma}{\alpha-c \gamma}=N_{03} .
$$

With set of parameter values as described in Section 5, one can find out estimated length of stability preserving delays as $T_{1}^{+}=2.2137$ and $T_{2}^{+}=0.7808$, respectively, with the choice $F_{1}=F_{2}=0.5$. Figures 6.1 and 6.2 depict the solution of delayed model system (5.1) for the situations $T_{1}<T_{1}^{+}, T_{2}<T_{2}^{+}$and $T_{1}>T_{1}^{+}, T_{2}>T_{2}^{+}$, respectively.

If $A_{1}$ is very small, $T_{1}^{+}$will be very large. It is possible to find out the limiting value of $T_{1}^{+}$as $A_{1} \rightarrow 0$ but we are not interested with that particular limit. We like to comment that when magnitude of $A_{1}$ is small, then the time delays with large magnitude will not alter the stability property of coexisting equilibrium point. Hence in that case large delay will become harmless for stability of coexisting equilibrium point. Hence we can conclude with the condition (6.26), namely, "the constant input of detritus is less than the critical value $N_{03}$ " that if the sum of two delays, the nutrient recycling delay, and the gestation delay due to consumption of detritus by the detritivores is very large, the model system (5.1) with $E_{*}$ as interior equilibrium will remain stable. Since $A_{2}$ is positive, the estimate $T_{2}^{+}$is valid. If $A_{2}$ is very small, then $T_{2}^{+}$is very large. Hence we can conclude that if the delay in conversion of the detritivores to predators is very large, then the equilibrium point $E_{*}$ will remain stable.

Let us suppose that the sum of two delays, recycling delay of the detritus and the delay due to gestation of the detritivores, is equal to the delay due to gestation of the predators 


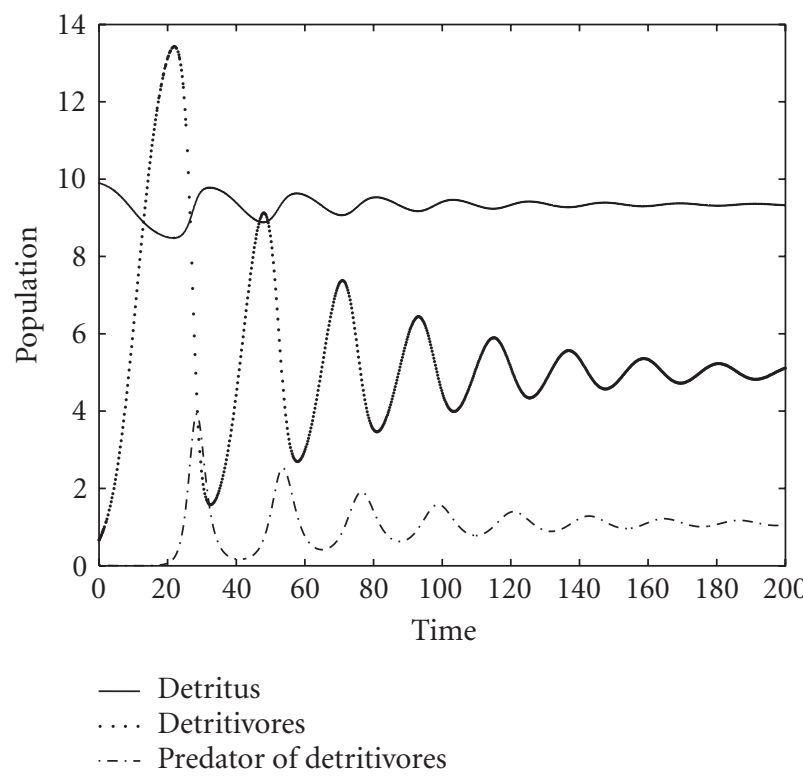

Figure 6.1. Solution of (5.1) with $T_{1}=2.1$ and $T_{2}=.7$, other parameters are same as described in Section 5 .
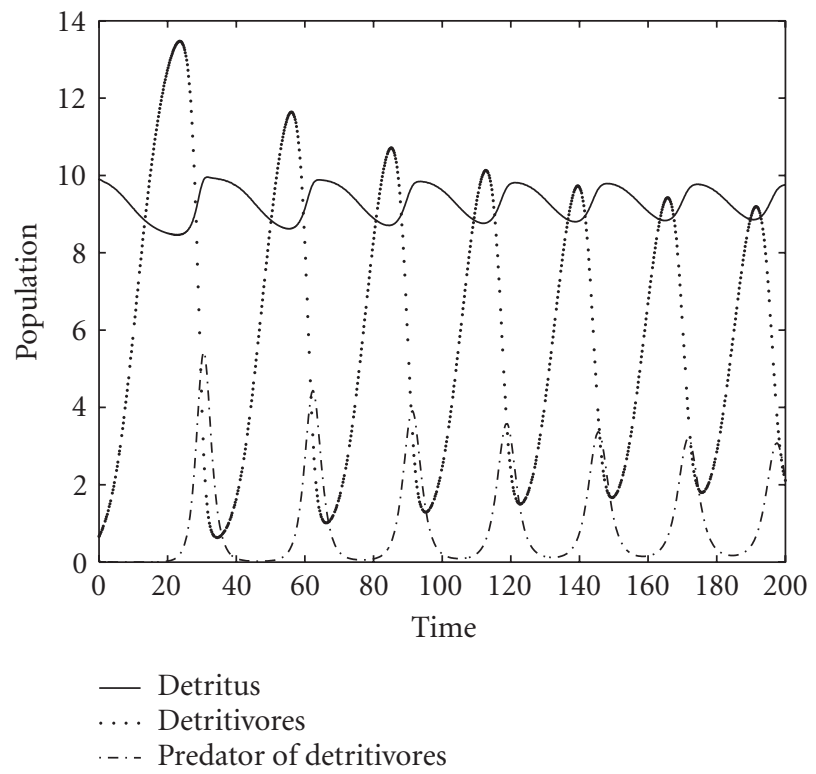

Figure 6.2. Solution of (5.1) with $T_{1}=2.5>T_{1}^{+}$and $T_{2}=.8>T_{2}^{+}$, other parameters are same as described in Section 5. 
of detritivores, that is, $T_{1}=T_{2}=T$, then (6.17) reduces to

$$
\begin{aligned}
& {\left[\frac{\rho w}{p} \sin w T+\rho(1-\cos w T)\right]+\left[\left|\frac{\phi w}{p}+\frac{r}{w}\right| \sin w T+\left|\phi-\frac{r}{p}\right|(1-\cos w T)\right]} \\
& <\theta+\rho+\phi-\frac{r}{p}=\eta
\end{aligned}
$$

or, equivalently,

$$
\left[(\rho+\phi) \frac{w}{p}+\frac{r}{w}\right] \sin w T+\left(\rho+\phi-\frac{r}{p}\right)(1-\cos w T)<\theta+\rho+\phi-\frac{r}{p}=\eta .
$$

Let us denote the left-hand side of $(6.28)$ by $\Theta(w, T)$. The trigonometric inequalities $\sin w T \leq w T$ and $1-\cos w T \leq(1 / 2) w^{2} T^{2}$ reduce $(6.28)$ to

$$
\Theta(w, T) \leq\left[(\rho+\phi) \frac{w^{2}}{p}+r\right] T+\left|\rho+\phi-\frac{r}{p}\right| \frac{1}{2} w^{2} T^{2} \equiv \Phi(T, w) .
$$

For $0<w<w_{+}, \Theta(T, w) \leq \Phi(T, w) \leq \Phi\left(T, w_{+}\right)$.

Now if $\Phi\left(T, w_{+}\right)<\eta$, then also $\Theta\left(T, w_{0}\right)<\eta$. Let us now suppose that $\Phi\left(T, w_{+}\right)=\eta$, then

$$
A T^{2}+B T-\eta=0
$$

where

$$
A=\frac{1}{2}\left|\rho+\phi-\frac{r}{p}\right| w_{+}^{2}, \quad B=(\rho+\phi) \frac{w_{+}^{2}}{p}+r .
$$

Therefore,

$$
T^{+}=\frac{1}{2 A}\left[-B+\sqrt{B^{2}+4 A \eta}\right]
$$

Thus for $0<T<T^{+}$, the Nyquist criteria hold. $T^{+}$gives estimate for the length of delay for which stability is preserved. Here $T^{+}$is dependent on the expression of $A$ in (6.31) which is again function of the system parameters. Hence we can conclude that the estimate for the delay is totally dependent on the system parameters for which the interior equilibrium $E_{*}$ is locally asymptotically stable.

\section{Bifurcation analysis}

In this section we try to find condition (by considering the discrete time delays $\tau_{1}, \tau_{2}$, $\tau_{3}$ as a bifurcation parameter) under which the models (5.1a)-(5.1c) exhibit small amplitude oscillation arising from Hopf-bifurcation [16, 24]. For this purpose we recall the characteristic equation (5.5)

$$
\Delta\left(\lambda, T_{1}, T_{2}\right)=\lambda^{3}+p \lambda^{2}+\left[\theta+\rho e^{-\lambda T_{1}}+\phi e^{-\lambda T_{2}}\right] \lambda+r e^{-\lambda T_{2}}=0
$$


as we are interested with bifurcation analysis around the interior equilibrium point $E_{*}$ for the variation of delay parameter. The stability of the equilibrium $E_{*}$ is determined by the sign of real part of the characteristic root of (7.1).

By our assumption $\lambda$ is a function of time delays $\tau_{1}, \tau_{2}, \tau_{3}$, if we write $\lambda=\mu+i \nu$, then $\mu$ and $\nu$ are also functions of $\tau_{1}, \tau_{2}, \tau_{3}$, that is, $\mu \equiv \mu\left(\tau_{1}, \tau_{2}, \tau_{3}\right) \equiv \mu\left(T_{1}, T_{2}\right), \nu \equiv \nu\left(\tau_{1}, \tau_{2}, \tau_{3}\right) \equiv$ $\nu\left(T_{1}, T_{2}\right)$. Substituting

$$
\lambda\left(\tau_{1}, \tau_{2}, \tau_{3}\right) \equiv \lambda\left(T_{1}, T_{2}\right)=\mu\left(T_{1}, T_{2}\right)+i \nu\left(T_{1}, T_{2}\right)
$$

in (7.1) and then separating real and imaginary parts, we obtain

$$
\begin{gathered}
\mu^{3}-3 \mu \nu^{2}+p\left(\mu^{2}-\nu^{2}\right)+\left[\theta+\rho e^{-\mu T_{1}} \cos \nu T_{1}+\phi e^{-\mu T_{2}} \cos \nu T_{2}\right] \mu \\
+\left[\rho e^{-\mu T_{1}} \sin \nu T_{1}+\phi e^{-\mu T_{2}} \sin \nu T_{2}\right] \nu+r e^{-\mu T_{2}} \cos \nu T_{2}=0, \\
-\nu^{3}+3 \mu^{2} \nu+2 p \mu \nu+\left[-\rho e^{-\mu T_{1}} \sin \nu T_{1}-\phi e^{-\mu T_{2}} \sin \nu T_{2}\right] \mu \\
+\left[\theta+\rho e^{-\mu T_{1}} \cos \nu T_{1}+\phi e^{-\mu T_{2}} \cos \nu T_{2}\right] \nu-r e^{-\mu T_{2}} \sin \nu T_{2}=0 .
\end{gathered}
$$

A necessary condition for the change of stability near the interior equilibrium point $E_{*}$ is that the characteristic equation (7.1) has purely imaginary roots. Since $\lambda$ as well as $\mu$ and $v$ are functions of $T_{1}$ and $T_{2}$, the change of stability behaviour occurs at such values of $T_{1}$ and $T_{2}$ such that $\mu\left(T_{1}, T_{2}\right)=0$ and $\nu\left(T_{1}, T_{2}\right) \neq 0$. Let $\widehat{T}_{1}$ and $\hat{T}_{2}$ be the critical values of $T_{1}$ and $T_{2}$, respectively, such that $\mu\left(\widehat{T}_{1}, \widehat{T}_{2}\right)=0$ and $\nu\left(\widehat{T}_{1}, \widehat{T}_{2}\right) \equiv \hat{\nu} \neq 0$. Then (7.3) become

$$
\begin{gathered}
-p \hat{\nu}^{2}+\left[\rho \sin \hat{\nu} \widehat{T}_{1}+\phi \sin \hat{\nu} \hat{T}_{2}\right] \hat{\nu}+r \cos \hat{\nu} \widehat{T}_{2}=0, \\
-\hat{\nu}^{3}+\left[\theta+\rho \cos \hat{\nu} \widehat{T}_{1}+\phi \cos \hat{\nu} \widehat{T}_{2}\right] \hat{\nu}-r \sin \hat{\nu} \widehat{T}_{2}=0 .
\end{gathered}
$$

Now we study the change of stability behaviour near the interior equilibrium $E_{*}$ when the parameters $T_{1}$ and $T_{2}$ cross through their critical values $\widehat{T}_{1}$ and $\widehat{T}_{2}$. First we study the change of stability behaviour near the interior equilibrium $E_{*}$ with respect to $T_{1}$. For this purpose we suppose that the other time delay $T_{2}$ is fixed at its critical value $\widehat{T}_{2}$.

From (7.4), eliminating $\widehat{T}_{1}$ and then rearranging we arrive at

$$
\begin{aligned}
\hat{\nu}^{6}+ & \left(p^{2}-2 \theta-2 \phi \cos \hat{\nu} \widehat{T}_{2}\right) \hat{\nu}^{4}-2(p \phi-r) \hat{\nu}^{3} \sin \hat{\nu} \widehat{T}_{2} \\
& +\left(\theta^{2}+\phi^{2}-\rho^{2}-2 p r \cos \hat{\nu} \widehat{T}_{2}+2 \theta \phi \cos \hat{\nu} \widehat{T}_{2}\right) \hat{\nu}^{2}-r \theta \hat{v} \sin \hat{\nu} \hat{T}_{2}+r^{2}=0 .
\end{aligned}
$$

Equation (7.5) is a transcendental equation, the complicated form of this equation is an obstruction to predict the nature of roots. Without going into a detailed analysis with (7.5) we assume there exists at least one real positive root of (7.5) and is denoted by $\hat{v}$. At this point we want to remark that the nonexistence of such a real root terminates our forthcoming bifurcation analysis, so far as our knowledge is concern no important ecological conclusion can be drawn based upon these mathematical outcome. The rest of the part is based upon the assumption that " $\hat{\nu}$ " is a positive real root of (7.5). Now, from 
(7.4) we can find the critical delay parameter $\widehat{T}_{1}$ for $\nu=\hat{v}$ as

$$
\widehat{T}_{1}=\frac{1}{\hat{v}} \arctan \left[\frac{p \hat{\nu}^{2}-\phi \hat{v} \sin \hat{\nu} \widehat{T}_{2}-r \cos \hat{\nu} \widehat{T}_{2}}{\hat{\nu}^{3}-\theta \hat{v}-\phi \hat{v} \cos \hat{\nu} \widehat{T}_{2}+r \sin \hat{\nu} \widehat{T}_{2}}\right]+\frac{n \pi}{\hat{v}}, \quad \text { where } n=0,1,2,3, \ldots
$$

One possible value of $\widehat{T}_{1}$ denoted by $\widehat{T}_{1}^{0}$ can be obtained from (7.6) for the choice $n=0$ as follows:

$$
\widehat{T}_{1}^{0}=\frac{1}{\hat{v}} \arctan \left[\frac{p \hat{\nu}^{2}-\phi \hat{v} \sin \hat{\nu} \hat{T}_{2}-r \cos \hat{\nu} \hat{T}_{2}}{\hat{\nu}^{3}-\theta \hat{v}-\phi \hat{\nu} \cos \hat{\nu} \hat{T}_{2}+r \sin \hat{\nu} \widehat{T}_{2}}\right]
$$

The expressions in (7.5) and (7.7) give critical values $\hat{v}$ and $\widehat{T}_{1}$ for which the characteristic root of (7.1) will have a pair of purely imaginary roots.

To verify the transversality condition of Hopf-bifurcation, we examine the value of $d \mu / d T_{1}$ evaluated at $T_{1}=\widehat{T}_{1}$ with the condition that $\mu\left(\widehat{T}_{1}\right)=0$ and $\nu\left(\widehat{T}_{1}\right) \equiv \hat{v} \neq 0$. If $d \mu / d T_{1}$ is a nonvanishing quantity, stabilization cannot take place at the critical parametric value $\widehat{T}_{1}$. We differentiate (7.4) with respect to $T_{1}$ and putting $T_{1}=\widehat{T}_{1}$ (using $\mu\left(\widehat{T}_{1}\right)=0$ and $\left.\nu=\hat{v}\right)$ we get

$$
\begin{aligned}
& A\left[\frac{d \mu}{d T_{1}}\right]_{T_{1}=\widehat{T}_{1}}+B\left[\frac{d v}{d T_{1}}\right]_{T_{1}=\widehat{T}_{1}}=C, \\
& -B\left[\frac{d \mu}{d T_{1}}\right]_{T_{1}=\widehat{T}_{1}}+A\left[\frac{d v}{d T_{1}}\right]_{T_{1}=\widehat{T}_{1}}=D,
\end{aligned}
$$

where

$$
\begin{aligned}
& A=-3 \hat{\nu}^{2}+\theta+\rho \cos \hat{\nu} \widehat{T}_{1}+\phi \cos \hat{\nu} \widehat{T}_{2}-\left[\rho \widehat{T}_{1} \sin \hat{\nu} \widehat{T}_{1}+\phi \widehat{T}_{2} \sin \hat{\nu} \widehat{T}_{2}\right] \hat{\nu}-r \widehat{T}_{2} \cos \hat{\nu} \widehat{T}_{2}, \\
& B=-2 p \hat{\nu}+\rho \sin \hat{\nu} \widehat{T}_{1}+\phi \sin \hat{\nu} \hat{T}_{2}+\left[\rho \widehat{T}_{1} \cos \hat{\nu} \widehat{T}_{1}+\phi \widehat{T}_{2} \cos \hat{\nu} \widehat{T}_{2}\right] \hat{\nu}-r \widehat{T}_{2} \sin \hat{\nu} \widehat{T}_{2}, \\
& C=-\rho \hat{\nu}^{2} \cos \hat{\nu} \widehat{T}_{1}, \quad D=\rho \hat{\nu}^{2} \sin \hat{\nu} \widehat{T}_{1}
\end{aligned}
$$

Solving (7.8) for $\left[d \mu / d T_{1}\right]_{T_{1}=\widehat{T}_{1}}$ one can obtain

$$
\left[\frac{d \mu}{d T_{1}}\right]_{T_{1}=\widehat{T}_{1}}=\frac{A C-B D}{A^{2}+B^{2}} .
$$

Clearly, the sign of $\left[d \mu / d T_{1}\right]_{T_{1}=\widehat{T}_{1}}$ is same as that of $A C-B D$. Therefore, we calculate

$$
\begin{aligned}
A C-B D= & -\rho \hat{\nu}^{2}\left[\left(\theta-3 \hat{\nu}^{2}\right) \cos \hat{\nu} \widehat{T}_{1}-2 p \hat{\nu} \sin \hat{\nu} \widehat{T}_{1}+\rho+\phi \cos \hat{\nu}\left(\widehat{T}_{1}-\widehat{T}_{2}\right)\right] \\
& -\rho \hat{\nu}^{2}\left[\phi \hat{\nu} T_{2} \sin \hat{\nu}\left(\widehat{T}_{1}-\widehat{T}_{2}\right)-r \widehat{T}_{2} \cos \hat{\nu}\left(\widehat{T}_{1}-\widehat{T}_{2}\right)\right] .
\end{aligned}
$$


Now, for values of $p, \theta, \rho, \phi, r$ and with (7.10) and (7.11) we see that $\left[d \mu / d T_{1}\right]_{T_{1}=\widehat{T}_{1}} \neq 0$ and consequently the transversality condition of Hopf-bifurcation is satisfied for $T_{1}=\widehat{T}_{1}^{0}$ which is given in (7.7). Thus the model system exhibits Hopf-bifurcating small amplitude periodic solutions as $T_{1}$ passes through its critical value $\widehat{T}_{1}^{0}$.

Similar study may be carried out by considering $T_{2}$ as a variable parameter. Now we consider the case when both the parameters are equal, that is, $T_{1}=T_{2}=T$. This may lead the study of the behaviour of stability change to some specific condition on the parameters of the model system. For the choice $T_{1}=T_{2}=T$, (7.3) become

$$
\begin{gathered}
\mu^{3}-3 \mu \nu^{3}+p\left(\mu^{2}-\nu^{2}\right)+\left[\theta+(\rho+\phi) e^{-\mu T} \cos \nu T\right] \mu \\
+(\rho+\phi) \nu e^{-\mu T} \sin \nu T+r e^{-\mu T} \cos \nu T=0, \\
-\nu^{3}+3 \mu^{2} \nu+2 p \mu \nu-\mu(\rho+\phi) e^{-\mu T} \sin \nu T+\left[\theta+(\rho+\phi) e^{-\mu T} \cos \nu T\right] \nu-r e^{-\mu T} \sin \nu T=0 .
\end{gathered}
$$

Now the characteristic root $\lambda$ as well as $\mu$ and $\nu$ are functions of $T$ only. To analyze the change of stability behaviour around $E_{*}$ with respect to $T$, it occurs at a particular value of $T$ for which $\mu(T)=0$ and $\nu(T) \neq 0$. Let $\hat{T}$ be the critical value of $T$ such that $\mu(\hat{T})=0$ and $\nu(\hat{T})=\hat{\nu} \neq 0$. Then (7.12) become

$$
\begin{gathered}
-p \hat{\nu}^{2}+(\rho+\phi) \hat{\nu} \sin \hat{\nu} \hat{T}+r \cos \hat{\nu} \hat{T}=0, \\
-\hat{\nu}^{3}+\theta \hat{\nu}+(\rho+\phi) \hat{\nu} \cos \hat{\nu} \hat{T}-r \sin \hat{\nu} \hat{T}=0 .
\end{gathered}
$$

$\widehat{T}$ eliminant of (7.13) give

$$
\hat{\nu}^{6}+\left(p^{2}-2 \theta\right) \hat{\nu}^{4}+\left[\theta^{2}-(\rho+\phi)^{2}\right] \hat{v}^{2}-r^{2}=0
$$

This is a cubic equation in $\hat{v}^{2}$. Here $p^{2}-2 \theta=\left(a_{11}-a_{22}\right)^{2}$ is always positive and the product of the roots is positive. Hence the equation has at least one positive root independent of the sign of $\theta^{2}-(\rho+\phi)^{2}$. A positive real root of (7.14) gives the expression for $\nu=\hat{v}$. For this expression of $\hat{v}$ we can find the expression for $T=\hat{T}$ from (7.13). Thus the critical parametric value $\hat{T}$ is given by

$$
\widehat{T}_{n}=\frac{1}{\hat{v}} \arctan \left[\frac{p(\rho+\phi) \hat{\nu}^{2}-r\left(\hat{\nu}^{2}-\theta\right)}{(\rho+\phi)\left(\hat{\nu}^{2}-\theta\right) \hat{\nu}+p r \hat{v}}\right]+\frac{n \pi}{\hat{\nu}}, \quad \text { where } n=0,1,2,3, \ldots
$$

One possible value of $\widehat{T}$ denoted by $\widehat{T}^{0}$ is obtained by choosing $n=0$ as

$$
\widehat{T}^{0}=\frac{1}{\hat{v}} \arctan \left[\frac{p(\rho+\phi) \hat{\nu}^{2}-r\left(\hat{\nu}^{2}-\theta\right)}{(\rho+\phi)\left(\hat{\nu}^{2}-\theta\right) \hat{v}+p r \hat{v}}\right]
$$


Now, to analyze the change in stability behaviour around $E_{*}$ with respect to $T$, we examine the sign of $d \mu / d T$ when $\mu(\widehat{T})=0$ and $\nu=\hat{\nu} \neq 0$. If the value of the derivative is not zero, then clearly stability cannot take place at $T=\widehat{T}^{0}$. We differentiate (7.12) with respect to $T$ and then put $T=\widehat{T}^{0}, \mu=0$, and $\nu=\hat{\nu} \neq 0$, which gives

$$
P\left[\frac{d \mu}{d T}\right]_{T=\widehat{T}^{0}}+Q\left[\frac{d \nu}{d T}\right]_{T=\widehat{T}^{0}}=R, \quad-Q\left[\frac{d \mu}{d T}\right]_{T=\widehat{T}^{0}}+P\left[\frac{d \nu}{d T}\right]_{T=\widehat{T}^{0}}=S,
$$

where

$$
\begin{gathered}
P=-3 \hat{\nu}^{2}+\theta+(\rho+\phi)\left(\cos \hat{\nu} \widehat{T}^{0}-\hat{\nu} \widehat{T}^{0} \sin \hat{\nu} \widehat{T}^{0}\right)-r \widehat{T}^{0} \cos \hat{\nu} \widehat{T}^{0}, \\
Q=-2 p \hat{\nu}+(\rho+\phi)\left(\sin \hat{\nu} \widehat{T}^{0}+\hat{\nu} \widehat{T}^{0} \cos \hat{\nu} \widehat{T}^{0}\right)-r \widehat{T}^{0} \sin \hat{\nu} \widehat{T}^{0}, \\
R=-(\rho+\phi) \hat{\nu}^{2} \cos \hat{\nu} \widehat{T}^{0}+r \hat{\nu} \sin \hat{\nu} \widehat{T}^{0}, \quad S=(\rho+\phi) \hat{\nu}^{2} \sin \hat{\nu} \widehat{T}^{0}+r \hat{\nu} \cos \hat{\nu} \widehat{T}^{0} .
\end{gathered}
$$

Solution of (7.17) for $[d \mu / d T]_{T=\widehat{T}^{0}}$ gives

$$
\left[\frac{d \mu}{d T}\right]_{T=\widehat{T}^{0}}=\frac{P R-Q S}{P^{2}+Q^{2}} .
$$

The sign of $[d \mu / d T]_{T=T^{0}}$ is same as $P R-Q S$. Using (7.13) and expressions (7.18) in (7.19), we obtain

$$
P R-Q S=\hat{v}^{2}\left[3 \hat{v}^{4}+2\left(p^{2}-2 \theta\right) \hat{v}^{2}+\theta^{2}-(\rho+\phi)^{2}\right]
$$

Now

$$
\left[\frac{d \mu}{d T}\right]_{T=\widehat{T}^{0}} \neq 0 \quad \text { if } 3 \hat{\nu}^{4}+2\left(p^{2}-2 \theta\right) \hat{\nu}^{2}+\theta^{2}-(\rho+\phi)^{2} \neq 0 .
$$

An expression for $\hat{v}^{2}$ can be obtained from the biquadratic equation $3 \widehat{v}^{4}+2\left(p^{2}-2 \theta\right) \hat{v}^{2}+$ $\theta^{2}-(\rho+\phi)^{2}=0$ as follows:

$$
\hat{v}^{2}=\frac{1}{3}\left[-\left(p^{2}-2 \theta\right)+\sqrt{\left(p^{2}-2 \theta\right)^{2}-3\left\{\theta^{2}-(\rho+\phi)^{2}\right\}}\right]=\hat{v}_{0}^{2},
$$

where $\theta^{2}-(\rho+\phi)^{2}<0$ due to the condition $p^{2}-2 \theta>0$. Thus

$$
\begin{gathered}
{\left[\frac{d \mu}{d T}\right]_{T=\widehat{T}^{0}} \neq 0 \quad \text { if } \hat{v}^{2} \neq \hat{v}_{0}^{2} \text { for } \theta^{2}-(\rho+\phi)^{2}<0,} \\
\text { i.e., when }\left|a_{11} a_{22}\right|<\left|a_{12} a_{21}+a_{23} a_{32}\right| .
\end{gathered}
$$

If $T=0$, then $E_{*}$ is stable. According to Butler's lemmas [11,25], the negative real part of the eigenvalue remains negative for $T<\widehat{T}^{0}$, and therefore, $E_{*}$ remains stable. When $T=\widehat{T}^{0},[d \mu / d T]_{T=\widehat{T}^{0}} \neq 0$ if the condition (7.23) is satisfied. Then the real part of the eigenvalue becomes positive and the equilibrium point $E_{*}$ becomes unstable. When $T>$ $\widehat{T}^{0}$, the real part of the eigenvalue remains positive and the stabilization cannot take place as $T$ passes through its critical value $\widehat{T}^{0}$ and the solution bifurcates into Hopf-bifurcating small amplitude periodic solution around $E_{*}$. 


\section{Conclusion}

In this paper we have considered the dynamical behaviour of a homogeneous model of detritus-based ecosystem comprised with detritivores and predator of detritivores within deterministic environment. The present work is an extension of earlier work by Bandyopadhyay and Bhattacharya [2], where a simpler version of detritus-based ecosystem model is considered in absence of delay parameters. In the present study we have modified the model system by considering Holling type-II functional response for detritivores and predators of detritivores instead of mass action law. Discrete time delay is incorporated to study the effect of gestation delay and recycling delay on the dynamical behaviour of the model system. At this position we want to remark that no specific model can be set up to describe and understand the behaviours of natural systems like the detritus-based model system. Exposed discrepancies always suggest some modifications for the standing model. For this reason mathematical modelling of natural system is an evolving process.

Firstly we have stated and proved several results giving criteria for the existence of various equilibrium points, boundedness of solution, and local asymptotic stability condition of various equilibrium points of model system in absence of time delay. The most interesting and important result we have obtained for the delay differential equation model system is the estimated length of gestation delay and recycling delay which will preserve the stability of $E_{*}$. It is a well-known fact that the discrete time delays have ability to alter the dynamical behaviours significantly compared to the models described in terms of ordinary differential equation. Discrete time delay has the ability to drive a stable equilibrium point to an unstable one and it is also responsible for the oscillations of various trophic levels [2].

Before giving some ecological interpretations of the mathematical outcomes we want to remark that complex nature of the model system leads us some complicated mathematical results that are interesting from applied mathematics viewpoint but no conclusion can be drawn from ecological point of view. Ecological significance of various results obtained in this paper are as follows.

(i) Local stability analysis of the deterministic model (in absence of delay) reveals the fact that the constant input rate of detritus plays a crucial role behind the stability of coexisting equilibrium point and is also responsible for coexistence of detritivores and their predators. Food intake rate and conversion efficiencies have some significant contribution behind the dynamical behaviour of the model system.

(ii) For the present model system the stability of interior equilibrium point demands some extra criteria apart from its existence conditions which is a different result compared to the model considered in [2].

(iii) Inclusion of gestation delay and recycling delay into the model system produce some interesting dynamical behaviours. In Section 5, we have shown that the local asymptotic stability of $E_{*}$ must depend upon the length of time delays. It is impossible to find out stability condition of $E_{*}$ which will be independent of the magnitude of delay parameters. Numerically we have shown the change of stability behaviour with the increase in magnitude of discrete time delay. Conditions of local asymptotic stability of coexisting equilibrium point indicate that the longer time for gestation and recycling of biomass are responsible for instability of population density. 
(iv) In Section 6, we have obtained estimates for the length of delay, which will preserve the coexistence of individual species at their equilibrium levels. Length of gestation delays and recycling delay are related to the abundance of either species and detritus biomasses, consequently larger values of these parameters regulate the amount of food availability for other species. If the length of time delays were less than their estimated values, then the growth of detritivores and their predators are sufficient for their long time survival and also maintain the necessary amount of nutrients for higher trophic levels.

(v) Bifurcation analysis in Section 7 and the existence of Hopf-bifurcating periodic solution give some support behind the oscillating population density of individual species. Earlier model fails to capture this oscillatory behaviour of various species that is commonly observed in natural environment.

Finally, we want to remark that the complicated mathematical expressions involved with the analysis in Section 7 can be made simpler by considering some alternative mathematical models for detritus-based ecosystem. Verification of mathematical results with the help of practical data set will give better realization and in turn suggest some necessary modification for the modelling approach. At this moment we have no practical data set in our hand to verify the mathematical outcomes of the previous sections. Other types of functional responses and introduction of time delay in some different manners may give rise to better realization of biological phenomenon associated with detritus-based ecosystem, which remain an open problem for forthcoming days.

\section{Acknowledgments}

The authors are grateful to Professor C.G. Chakraborty, (S. N. Bose Professor of Theoretical Physics), Department of Applied Mathematics, University of Calcutta, for his continuous help and guidance throughout the preparation of the paper. The second author wishes to thank University Grant Commission, Government of India, for their financial support (Minor Research Project Grant no. F. PSW-048/04-05(ERO), dated 13.03.2005). Authors are also thankful to the learned referees for their valuable suggestions and helpful comments which have helped in better exposition of the paper.

\section{References}

[1] M. Bandyopadhyay, Global stability and bifurcation in a delayed nonlinear autotroph-herbivore model, Nonlinear Phenomena in Complex Systems 7 (2004), no. 3, 238-249.

[2] M. Bandyopadhyay and R. Bhattacharya, Non-linear bifurcation analysis of a detritus based ecosystem, Nonlinear Studies 10 (2003), no. 4, 357-372.

[3] E. Beretta and Y. Kuang, Convergence results in a well-known delayed predator-prey system, Journal of Mathematical Analysis and Applications 204 (1996), no. 3, 840-853.

[4] G. Birkhoff and G. C. Rota, Ordinary Differential Equation, Ginn, Massachusetts, 1982.

[5] F. Charles, Utilisation of fresh detritus derived from Cystoseira mediterranea and Posidonia oceania by deposit-feeding bivalve Abra ovata, Journal of Experimental Marine Biology and Ecology 174 (1993), no. 1, 43-64.

[6] J. M. Cushing, Integrodifferential Equations and Delay Model in Population Dynamics, Springer, Heidelberg, 1977.

[7] P. Das and A. B. Roy, Oscillations in delay differential equation model of reproductive hormones in men with computer simulations, Journal of Biological Systems 2 (1994), no. 1, 73-90. 
[8] M. C. Dash, Fundamentals of Ecology, Tata McGraw Hill, New Delhi, 2001.

[9] L. Debnath, Nonlinear Partial Differential Equations for Scientists and Engineers, Birkhüuser Boston, Massachusetts, 2005.

[10] M. A. Faust and R. A. Gulledge, Associations of microalgae and meiofauna in floating detritus at a mangrove Island, Twin Cays, Belize, Journal of Experimental Marine Biology and Ecology 197 (1996), no. 2, 159-175.

[11] H. I. Freedman and V. S. H. Rao, The trade-off between mutual interference and time lags in predator-prey systems, Bulletin of Mathematical Biology 45 (1983), no. 6, 991-1004.

[12] H. I. Freedman and P. Waltman, Persistence in models of three interacting predator-prey populations, Mathematical Biosciences 68 (1984), no. 2, 213-231.

[13] K. Gopalsamy, Harmless delays in model systems, Bulletin of Mathematical Biology 45 (1983), no. 3, 295-309.

[14] _ Delayed responses and stability in two-species systems, Journal of the Australian Mathematical Society. Series B. Applied Mathematics 25 (1984), no. 4, 473-500.

[15] Stability and Oscillations in Delay Differential Equations of Population Dynamics, Mathematics and Its Applications, vol. 74, Kluwer Academic, Dordrecht, 1992.

[16] B. D. Hassard, N. D. Kazarinoff, and Y. H. Wan, Theory and Applications of Hopf Bifurcation, London Mathematical Society Lecture Note Series, vol. 41, Cambridge University Press, Cambridge, 1981.

[17] A. Hastings, Delays in recruitment at different trophic levels: effects on stability, Journal of Mathematical Biology 21 (1984), no. 1, 35-44.

[18] I. R. Joint, Microbial production of an estuarine mudflat, Estuarine Coastal and Marine Science 7 (1978), no. 2, 185-195.

[19] S. E. Jorgensen, Energy and ecological system analysis, Complex Ecosystems (B. C. Pattern and S. E. Jorgensen, eds.), Prentice Hall, New York, 1994.

[20] Y. Kuang, Delay Differential Equations with Applications in Population Dynamics, Mathematics in Science and Engineering, vol. 191, Academic Press, Massachusetts, 1993.

[21] J. R. Linley, Studies on larval biology of Leptoconops (Kieff.)(Diptera: Ceratopogonidae), Bulletin of Entomological Research 58 (1968), no. 1, 1-24.

[22] J. R. Linley and G. M. Adams, Ecology and behaviour of immature Culicoides melleus(Coq.) (Diptera: Ceratogonidae), Bulletin of Entomological Research 62 (1972), no. 1, 113-127.

[23] N. MacDonald, Time Lags in Biological Models, Lecture Notes in Biomathematics, vol. 27, Springer, Berlin, 1978.

[24] J. E. Marsden and M. McCracken, The Hopf Bifurcation and Its Applications, Springer, New York, 1976.

[25] A. Martin and S. Ruan, Predator-prey models with delay and prey harvesting, Journal of Mathematical Biology 43 (2001), no. 3, 247-267.

[26] R. M. May, Time delay versus stability in population models with two and three trophic levels, Ecology 54 (1973), no. 2, 315-325.

[27] _ Stability and Complexity in Model Ecosystems, Princeton University Press, New Jersey, 2001.

[28] J. D. Murray, Mathematical Biology, Biomathematics, vol. 19, Springer, Berlin, 1993.

[29] S. Ray and A. Choudhury, Salinity tolerance of Culex sitiens (Weid.) (Diptera: Culicidae) larvae in laboratory condition, Current Science 57 (1988), no. 3, 159-160.

[30] S. Ruan, Absolute stability, conditional stability and bifurcation in Kolmogorov-type predator-prey systems with discrete delays, Quarterly of Applied Mathematics 59 (2001), no. 1, 159-173.

[31] A. K. Sarkar and D. Ghosh, Role of detritus in a general prey-predator model of Sunderban Estuary, India, Biosystems 44 (1997), no. 2, 153-160. 


\section{Effect of time delay on a detritus-based ecosystem}

[32] A. K. Sarkar, D. Mitra, and A. B. Roy, Stability of partially closed producer-consumer system via decomposer, Ganit. Journal of Bangladesh Mathematical Society 10 (1990), no. 1-2, 21-27.

[33] T. F. Thingstad and T. I. Langeland, Dynamics of a chemostat culture: the effect of a delay in cell response, Journal of Theoretical Biology 48 (1974), no. 1, 149-159.

[34] E. W. Vetter, Population dynamics of a dense assemblage of marine detritivores, Journal of Experimental Marine Biology and Ecology 226 (1998), no. 1, 131-161.

[35] V. Volterra, Lecons sur la Théorie Mathématique de la Lutte Pour la Vie, Gauthier-Villars, Paris, 1931.

Nurul Huda Gazi: Department of Applied Mathematics, University of Calcutta, 92 APC Road,

Kolkata 700 009, India

E-mail address: nursha@rediffmail.com

Malay Bandyopadhyay: Department of Mathematics, Scottish Church College, Kolkata 700 006, India

E-mail address: malay_ban@rediffmail.com 


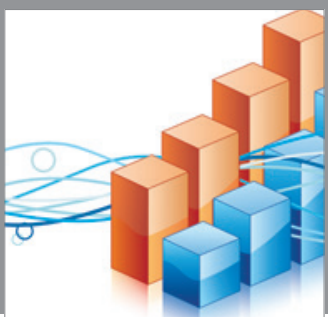

Advances in

Operations Research

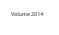

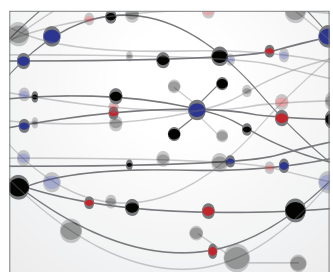

\section{The Scientific} World Journal
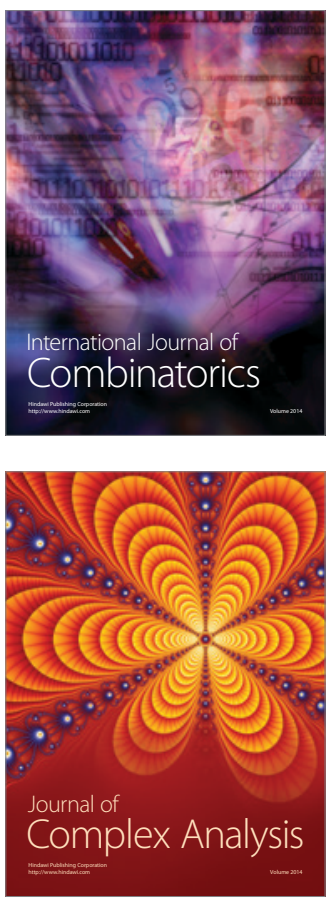

International Journal of

Mathematics and

Mathematical

Sciences
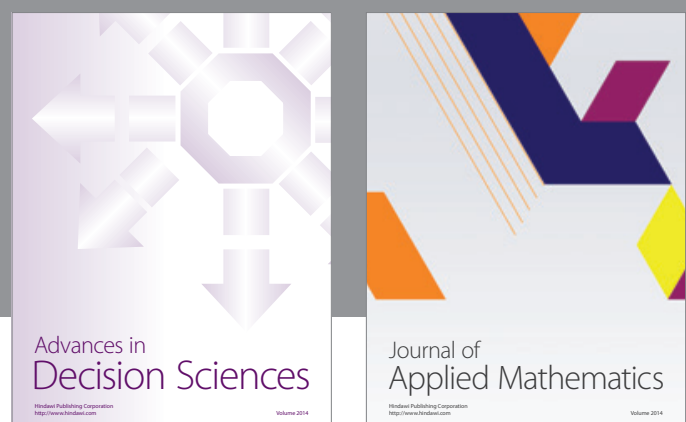

Journal of

Applied Mathematics
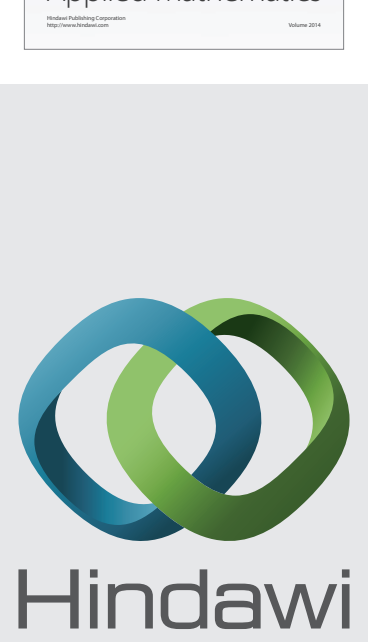

Submit your manuscripts at http://www.hindawi.com
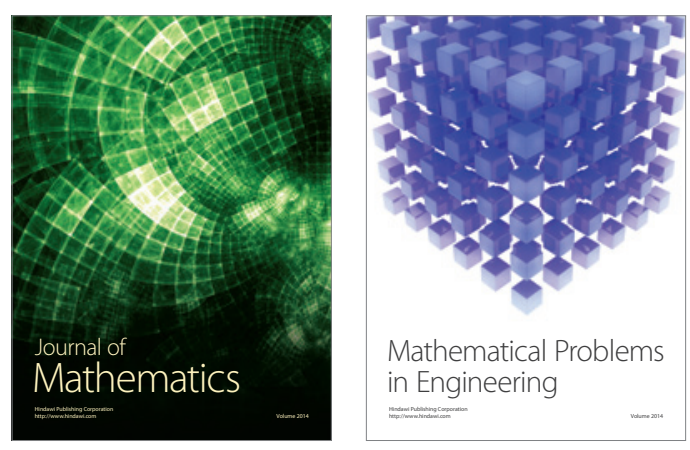

Mathematical Problems in Engineering
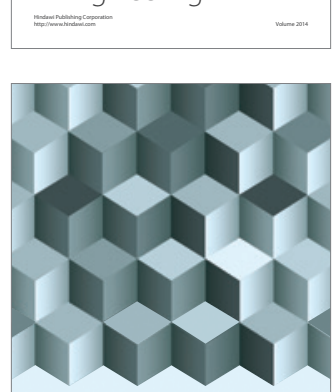

Journal of

Function Spaces
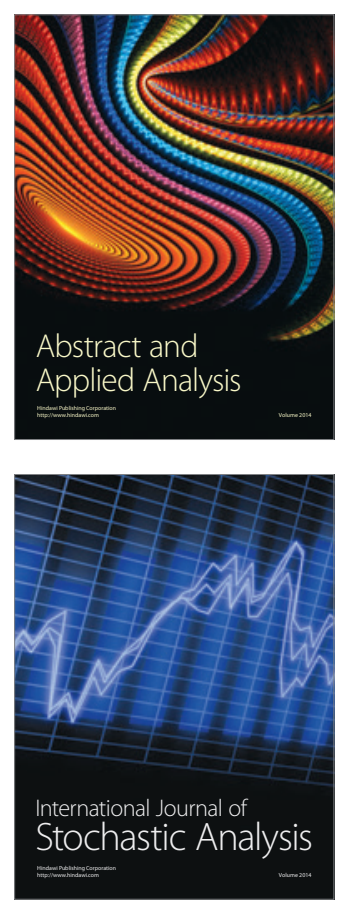

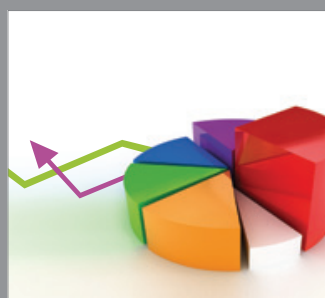

ournal of

Probability and Statistics

Promensencen
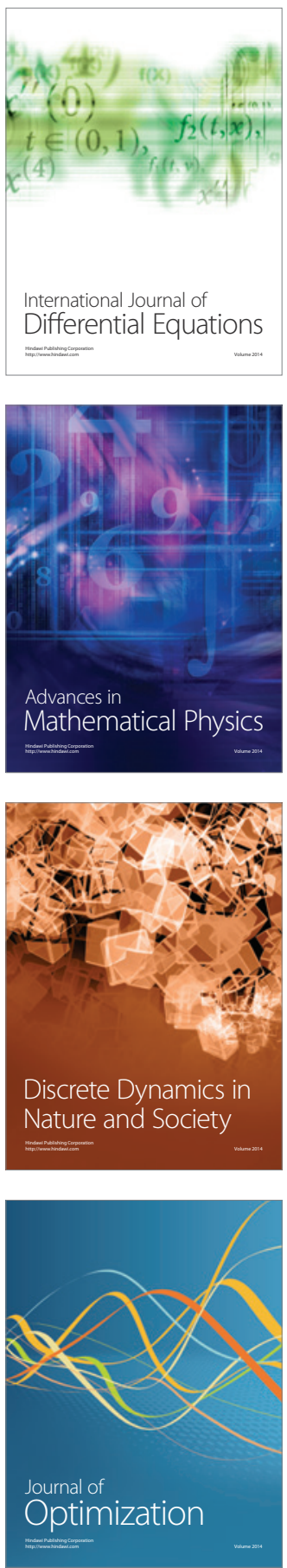